\title{
Wave Front Shearing Prism Interferometer
}

\author{
J. B. Saunders
}

(April 9, 1964)

\begin{abstract}
A small prism interferometer is described that is a modification of the Bates' wave front shearing interferometer. The prism is small, rugged, and versatile. The shape of any converging wave front from any optical element or compound instrument may be measured with this instrument.

Several variations of the prism are described that have characteristic properties. Special features are introduced for eliminating spherical aberration effects, for producing fringes of different characteristics, and for testing all wave fronts up to and exceeding that of an F.1 cone of rays.

Details for making and adjusting the prisms are given. Examples are given for interpreting the interferograms.
\end{abstract}

\section{Introduction}

The principles of the wave front shearing interferometer (WSI) have been described by Professor J. W. Bates [1]. This interferometer is particularly well suited for measuring the shapes of converging wave fronts. The difficulty of making the Bates instrument and others that have subsequently been described has limited the applications. This paper describes a simple form of WSI that is easily constructed, easily adjusted, and is practical. It is very small and can be mounted in an eyepiece cell for testing astronomical telescopes or as a convenient mount for other applications.

The WSI described by Bates consists of two beam dividers, two front-face mirrors, and two compensator plates. The angle of shear could be varied at will in the Bates model. Later models of the WSI by Drew [2] and Brown [3] are more compact. The Brown version is a relatively compact instrument of fixed shear. The instrument described here is more compact than any of the previous models, is quite easy to adjust, and is relatively free from vibration effects.

The WSI is quite suitable for testing lenses, mirrors, and combinations of these. It is also quite practical for studying atmospheric effects on light. Professor Bates described a method for analyzing the results from a WSI when the converging wave front is axially symmetrical. D. S. Brown [4] described another method of interpretation of sheared interferograms. The author of this paper has given a rigorous mathematical operation [5] for interpretation of sheared interferograms that does not depend upon symmetry of wave form. The solution is unique for any chosen "family" of reference points.

The elements forming this wave front shearing interferometer have been combined into a small compound prism that may be made into a variety of forms. Each form may be constructed with or without a built-in wedge that either permits or prevents rotation of the fringes. The two compo- nents of the prism are cemented together for stabilization of those adjustments that should remain fixed, without limiting the adjustments that are essential to its operation. The unit is small, rugged, compact, easily constructed, easily adjusted, and simple to use.

\section{Basic Optical Principles}

The wave front shearing interferometer (WSI) is a modification of that developed by Mach and Zehnder [6]. It differs from the MZ-interferometer in that convergent light is used instead of collimated light, the reflecting and beam-dividing surfaces are not parallel, the component beams emerge nonparallel, and the fringes are nonlocalized. The angle between the emerging components of any ray represents the angle of shear. To get observable interference between these two beams a source that is small [1] in the direction of shear is necessary.

The differential deviation of the component beams is accomplished in the Bates WSI by tilting one of the beam dividers, whereas in the Drew WSI it is effected by rotating one of the front-face mirrors. The vertex, or point of convergence, of one of the component wave fronts (images of the source) is always located near the optical element that is used to effect the differential deviation (angle of shear). The lateral separation of the two images of the source determines the width of the fringes (as in the Fresnel's mirrors [7] arrangement.)

In figure $1 \mathrm{~A}$, the lens, $L$, forms two images of the light source, $S$, at $S_{1}$ and $S_{2}$. The two component beams (represented by the two components of the principal ray and designated as 1 and 2) suffer total internal reflection at points $P_{1}$ and $P_{2}$. Each of these beams again divides at plane $B$ into two components - the transmitted beam, 1, dividing into beams $1^{\prime}$ and $1^{\prime \prime}$ and the reflected beams, 2, dividing into beams $2^{\prime}$ and $2^{\prime \prime}$. An observer's eye located close to the prism so as to receive either pair of rays $1^{\prime}$ and $2^{\prime \prime}$ or $1^{\prime \prime}$ and $2^{\prime}$ will be able to see two images of $L$ ( $L_{1}$ and $L_{2}$, figure $\left.1 \mathrm{~B}\right)$. 

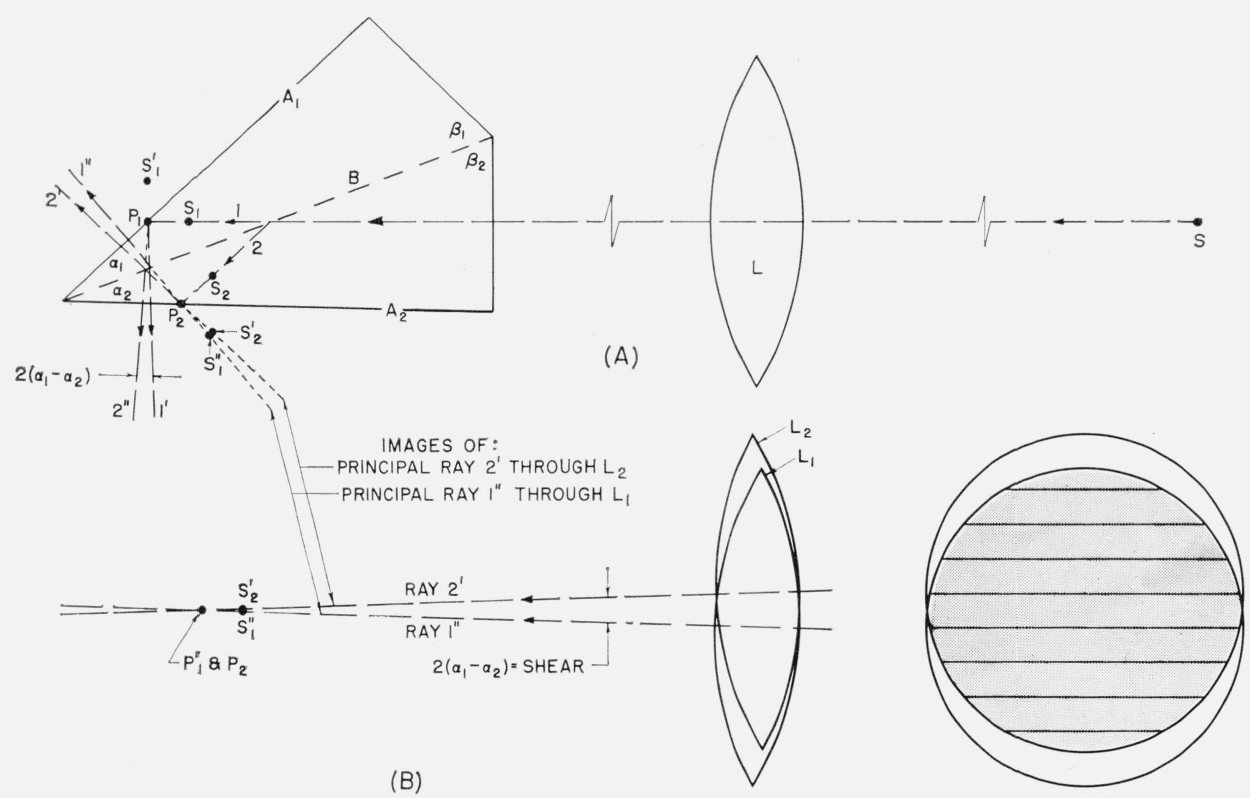

(C)

Figure 1A. Optical arrangement for testing a lens, $L$. The two elements of the prism, $A_{1} B$ and $A_{2} B$, are cemented together, with a semireflecting coat of aluminum on one of the cemented surfaces. Refraction at the two exit faces is assumed to be negligible.

B. $L_{1}$ and $L_{2}$ are images of $L$, as seen through prisms $A_{1} B$ and $A_{2} B$, respectively, by an observer's eye located behind the prism and receiving rays $1^{\prime \prime}$ and $2^{\prime}$. The image $P_{1}^{\prime}$ of $P_{1}$ appears to coincide with $P_{2}$.

C. Fringes of interference that appear to be localized in the overlapping areas of the two images, $L_{1}$ and $L_{2}$.

The two component prisms, shown in figure $1 \mathrm{~A}$, have angles $\alpha_{1}$ and $\alpha_{2}$ that differ by a chosen amount. Prism $A_{1} B$ is assumed to have been moved parallel to the plane of figure 1 and relative to prism $A_{2} B$ so that the image of $P_{1}\left[P_{2}\right]$, with respect to the semireflecting plane $B$, coincides with $P_{2}\left[P_{1}\right]$. This places $P_{1}$ and $P_{2}$ equal distances from both the source and the observer. The two emerging beams, $1^{\prime}$ and $2^{\prime \prime}$, appear to intersect at $P_{1}$ and the other pair of beams, $1^{\prime \prime}$ and $2^{\prime}$, appears to intersect at $P_{2}$. Images of $S_{1}$ and $S_{2}$ appear to be located at $S_{1}^{\prime \prime}$ and $S_{2}^{\prime}$, respectively. This adjustment of the prisms makes the two paths of light through the center of the lens equal so that when white light is used the zero order of interference is observed to pass midway between the centers of the two images of the lens. This fringe is almost colorless with a relatively pure colored fringe on each side of it. All other fringes are colored with varying tints due to overlapping of colors.

If the points $P_{1}$ and $P_{2}$ are further from $L$ than $S_{1}$ and $S_{2}$, images of $S$ are formed between the lens and these points. If, however, the points $P_{1}$ and $P_{2}$ are nearer the lens than $S_{1}$ and $S_{2}$, the images of $S$ are beyond the points $P_{1}$ and $P_{2}$. By moving either the prism, the lens, or the source along the axis of the lens, the emerging components of the principal rays remain fixed relative to the prism and the two component images of the source, $S_{1}^{\prime \prime}$ and $S_{2}^{\prime}$ (see figure $1 \mathrm{~B}$ ), appear to move along the two components of the principal ray. If the two images are made to coincide with $P_{1}^{\prime}$ and $P_{2}$ they will also coincide with each other and the two components, $1^{\prime \prime}$ and $2^{\prime}$ (or $1^{\prime}$ and $2^{\prime \prime}$ ), of the principal ray intersect at $P_{2}$ (or $\left.P_{1}^{\prime}\right)$. Consequently the lateral separation of the two coherent images, $S_{1}^{\prime \prime}$ and $S_{2}^{\prime}$, (figure $2 \mathrm{~A}$ ) may be varied at will by a simple linear translation of the prism along the axis of the lens.

Since the two images, $S_{1}$ and $S_{2}$, are coherent, of equal intensity, and equally distant from both the observer and the source, $S$, the two emerging cones of light will combine to produce low-order interference. The two coherent images act exactly as the two coherent sources in the Fresnel's mirrors [7] arrangement. The fringes of interference are nonlocalized. If either of the two pairs of beams, 1' and $2^{\prime \prime}$ or $1^{\prime \prime}$ and $2^{\prime}$, is projected onto a photographic emulsion (on film or paper) a photograph of the fringes is obtained. Also using a camera to receive either pair of beams, the fringes may be photographed in good contrast. The focusing of the camera does not vary the contrast, but if the lens, $L$, is imperfect the nature of the interferogram (fringe pattern) will vary with changes in the focus of the camera because the shape of the wave front changes as it is propagated from one position to another.

Interference fringes appear only in the overlapping areas (fig. 1C) of the two component beams. These fringes will be straight if the lens, $L$, is free from axial aberrations. Figure $2 \mathrm{~A}$ shows several sets of fringes that illustrate the effect of moving the prism along the axis of the lens. As in the Fresnel's mirrors arrangement, the fringes are always perpendic- 
ular to the direction of the straight line joining $S_{2}^{\prime}$ and $S_{1}^{\prime \prime}$ and their width (or separation) is inversely proportional to the lateral distance between the two images $S_{2}^{\prime}$ and $S_{1}^{\prime \prime}$.

Since the two rays, $1^{\prime \prime}$ and $2^{\prime}$, intersect (lying in a common plane) and since the two images, $S_{2}^{\prime}$ and $S_{1}^{\prime \prime}$, are equally distant from the point of intersection, the relative direction of the two images does not change. Consequently, the fringes do not rotate with movement of the prism along the principal ray.

If the compound prism (fig. 1A) is moved perpendicular to the axis of the lens and in the plane of figure $1 \mathrm{~A}$, the distance from the two images, $S_{2}^{\prime}$ and $S_{1}^{\prime \prime}$ (fig. 2B), to the observer becomes unequal and the zero order of interference moves away from the center of the field. The movements of the fringes, due to prism movements along the axis, will be similar to those in figure $2 \mathrm{~A}$ and the zero order of interference will be displaced from the center of the field. The zero-order fringe is indicated in figure 2 by representing it with lines that are heavier than for other orders.

The analysis of wave fronts by the method described in reference [5] requires that a "family of reference points" (appendix A) be chosen along a line parallel to the direction of shear.

If prisms $A_{1} B$ and $A_{2} B$ (fig. 3 ) are each rotated a few degrees about an axis normal to $B$ and through points $P_{1}$ and $P_{2}$, rays 1 and 2 will be deflected out of the plane of figure $3 \mathrm{~A}$. If the rotation is equal for the two prism components but in opposite directions the two component rays will be deflected by equal amounts. This results in a shearing of the wave front. The direction of shear is perpendicular to the plane of figure $3 \mathrm{~A}$, whereas the shear indicated by figure $1 \mathrm{~B}$ was in the plane of figure $1 \mathrm{~A}$. The two emerging rays $1^{\prime}$ and $2^{\prime \prime}$ (or $1^{\prime \prime}$ and $2^{\prime}$ ) lie in a plane that is normal to that of figure $3 \mathrm{~A}$. Figure $3 \mathrm{~B}$ shows the observed images of the lens as seen by beams $1^{\prime \prime}$ and $2^{\prime}$, in a plane normal to that of figure $3 \mathrm{~A}$. The plane of figure $3 \mathrm{~B}$ is also normal to that of figure $1 \mathrm{~B}$.

The two prisms shown in figures 1 and 3 shear the wave front in directions that are perpendicular and parallel, respectively, to the vertices of the prism angles. The spacing of the fringes in both prisms varies when the prism is moved along the principal ray. The fringes in both prisms move laterally, without change of spacing, when the prism is moved in the direction of shear and do not change for small movements of the prism perpendicular both to the principal ray and to the direction of shear.

If the prisms in figures 1 and 3 have been adjusted to place the images of $P_{1}$ and $P_{2}$ coincident, the two sources will always be equally distant from the intersection of the components of the principal rays. Consequently, the direction of the line joining the two image sources will not change and the interference fringes will not rotate. If the fringes are to be rotatable the components of the principal ray must not intersect each other.

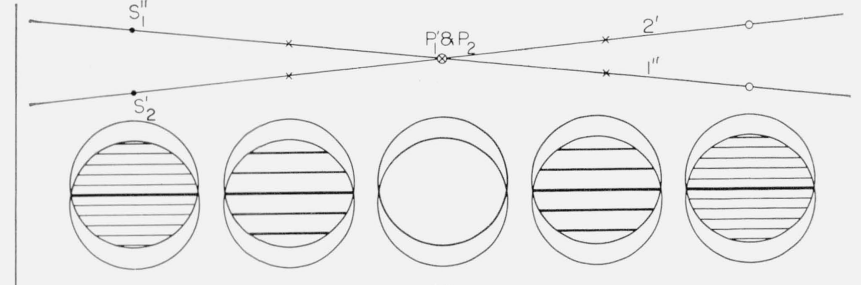

(A)

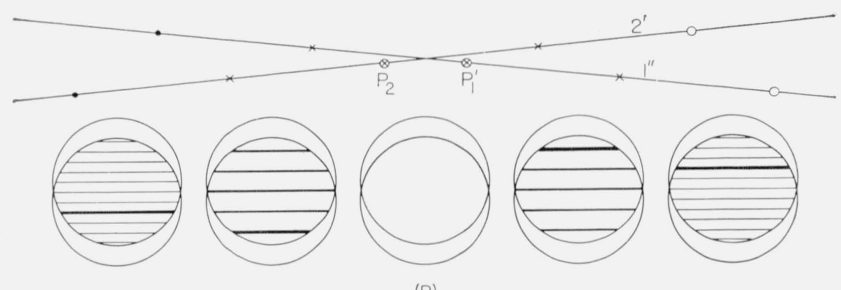

(B)

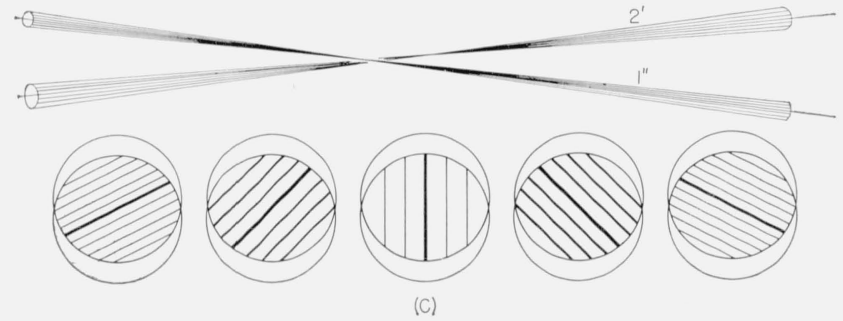

FIGURE 2A. The components of the principal ray $\left(1^{\prime \prime}\right.$ and $2^{\prime}$ of fig. $1 \mathrm{~B}$ ) intersect at coincident points $P_{1}^{\prime}$ and $P_{2}$. The fringe spacing varies with separation of images $S_{2}{ }^{\prime}$ and $S_{1}{ }^{\prime \prime}$ but do not rotate.

B. Components of principal ray intersect, but not at $P_{1}^{\prime}$ or $P_{2} . \quad P_{1}^{\prime}$ and $P_{2}$ are equally distant from the point of intersection. The zero-order fringe is displaced from the center of the pattern.

C. The two component rays, $1^{\prime \prime}$ and $2^{\prime}$ do not intersect. The horizontal component of the fringe width is invariant and the vertical component varies as in the above figures, $A$ and $B$. This results in a rotation of the fringes. The fringe width is a maximum when the separation of the two images of the source are closest together.

It is desirable, in many cases, to use white light sources for testing lenses-particularly for testing astronomical telescopes with light from celestial stars. When white light is used for the source the number of usable fringes is limited. In this case it is desirable that the fringes be as nearly parallel to the line of reference points as possible and with the zero-order fringe (the fringe of best visibility) located on or among the reference points. The prism described above and illustrated in figure 1 does not permit this adjustment without making the fringes too broad for accurate readings, unless the wave front has considerable distortion.

The WSI prism may be designed to permit rotation of the fringes. In the preferred design for rotatable fringes, the shearing of the component beams is accomplished by using two identical prism compo- 


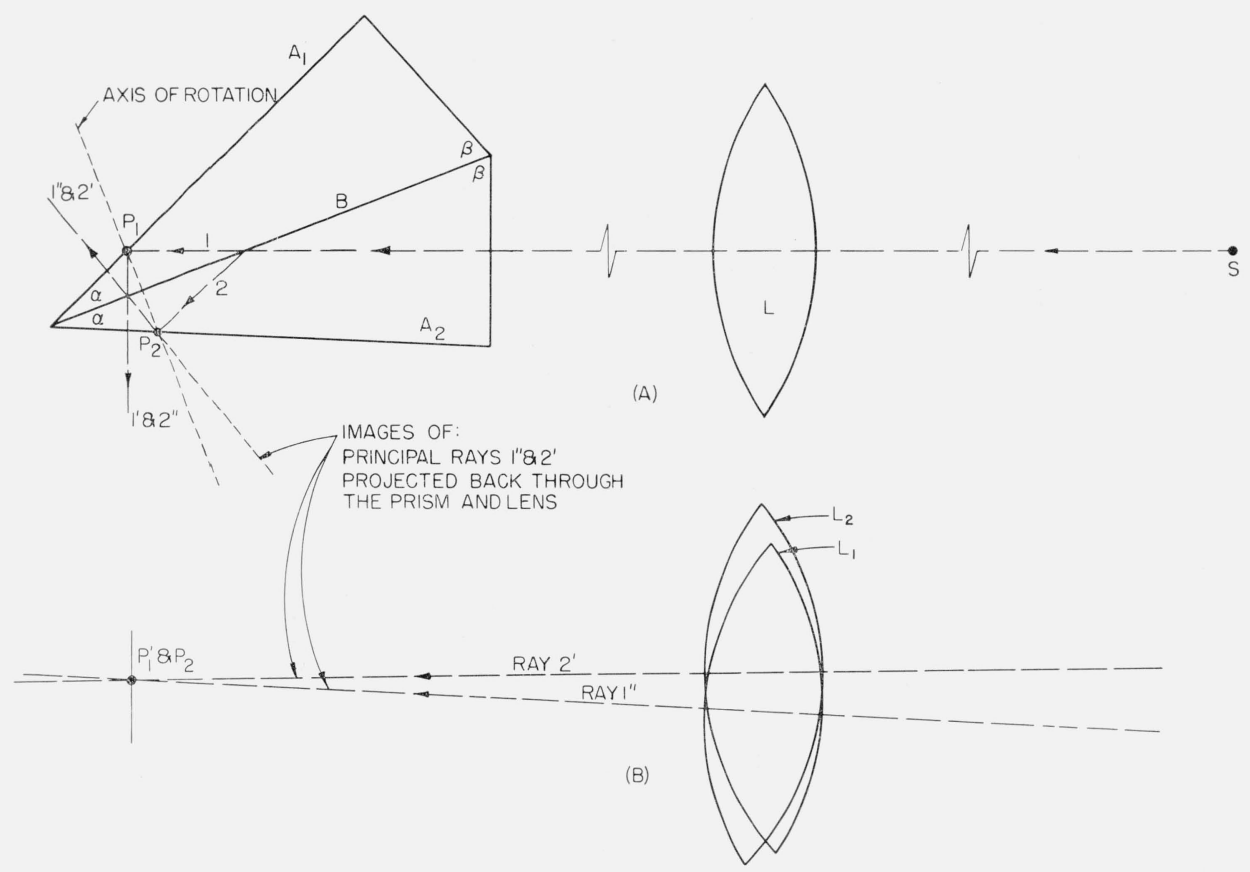

FIGURE 3A. The two components of the prism are identical, with the aluminum coat on one prism only. Shear is obtained by rotating the two components about an axis normal to $B$, but in opposite aireclions.

B. The planes of figures $A$ and $B$ are normal to each other.

nents with a relative rotation of these components about an axis that is normal to the dividing plane, $\mathrm{B}$. If the angles of the two component prisms are equal and no shear is introduced, the two component rays $1^{\prime}$ and $2^{\prime \prime}$ or $1^{\prime \prime}$ and $2^{\prime}$ (figure 3 ) will coincide. The two images of $L$ (shown in fig. $3 \mathrm{~B}$ as $L_{1}$ and $L_{2}$ ) will coincide. The optical path difference between rays 1 and 2, and all other rays in the beam, will be zero. No fringes will be observed because the phase difference is constant over the entire beam.

To obtain rotation of the fringes the two component rays $1^{\prime \prime}$ and $2^{\prime}$ (and also $1^{\prime}$ and $2^{\prime \prime}$ ) are shifted, relative to each other, in the direction normal to the plane of figure $3 \mathrm{~B}$. Figure 4 shows the ray trace through a prism that has been adjusted to perform this shift. Dividing planes $B_{1}$ and $B_{2}$ are parallel to each other and separated by a distance, $t$, that is proportional to the resultant lateral separation, $d$, of the two component emerging beams.

The prism of figure 4 is adjusted to produce zero optical path difference for the two component principal rays. Shear is obtained by a relative rotation of the prisms, exactly as described for the prism of figure 3 .

The two components, 1 and 2 , of the principal ray will emerge in planes that are perpendicular to the plane of figure 4 and separated by the distance $d$. The component rays will make equal angles, but of opposite sign, with the plane of figure 4 . When the prism is moved along the axis of the lens the two images of the source move along the two rays 1 and 2 , remaining equally distant from both the source and the observer. The zero-order fringe remains at the center of the field. The separation and relative direction of the two images of the source change with this movement of prism. Consequently, the fringes rotate and change width during this movement.

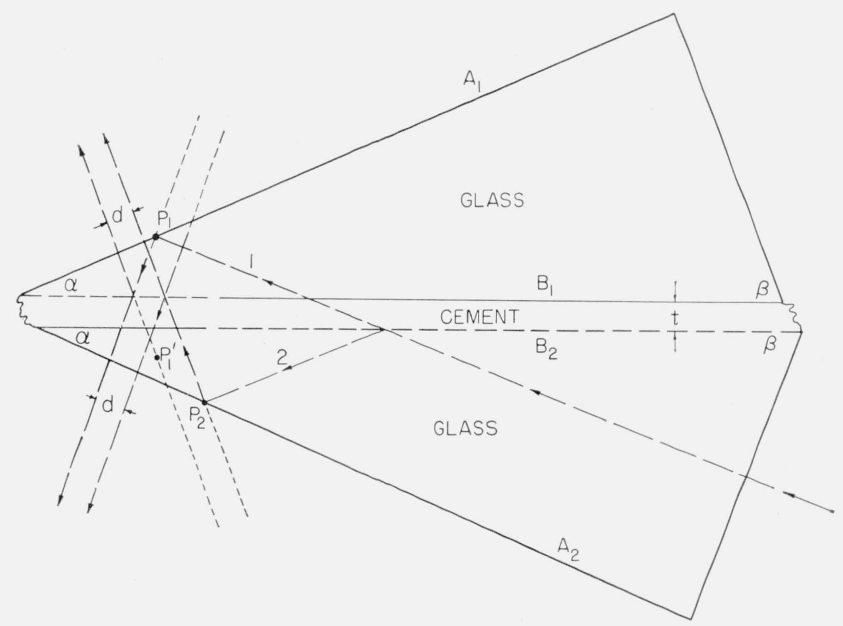

FIGURE 4. The two components of the prism are identical. The reflecting film on $R_{2}$ extends from the vertex of $\beta$ to the normal projection of $P_{2}$ onto $P$. The reflecting film on $P_{1}$ extends from the vertex of $\alpha$ to the normal projection of $P_{2}$ on $P_{1}$.

The uncoated parts of $B_{1}$ and $B_{2}$ virtually disappear if the index of the glass equals that of the cement. 
Figure $2 \mathrm{C}$ indicates the two pencils of rays about the component rays 1 and 2 . The direction and width of fringes for several positions of the prism, relative to the lens, are shown.

When the fringes are alined in the direction of shear they are of maximum width. Since the reference points must be on a line parallel to the direction of shear, the zero-order fringe can be alined with the line of reference points (assuming a good lens is being tested) and the zero-order fringe can be shifted laterally for most favorable reading of orders.

\section{Symbolic Designation of Prism Types}

The original prism interferometer was found to be a special case of a large variety of forms. Also, each form may be constructed and adjusted to provide a variety of properties, angles of shear, and other special adaptations. A description of one or two of these prisms would not adequately cover the development.

There are two geometrical forms, a quadrilateral and a hexagonal prism (see fig. 5). 'The usable angles of each of these two forms cover a considerable but finite range. Either form of prism with any combination of usable angles may be adjusted to have any angle of shear over a considerable range. There are two distinctive ways of producing shears which are normal to each other relative to the prism. Each of these types of shear is associated with adjustments of the interference fringes that have advantages and disadvantages for various types of measurements.

In order to describe these prisms adequately we will describe their common properties, method of adjustment, construction, etc., as a group. To do this an abbreviation will be used for designation of groups, and the abbreviations are so chosen that for future use definite specifications may be indicated for all types and forms. The chosen abbreviation is a compound letter-number symbol that indicates several of the prism's significant characteristics. The generalized symbol is NEWS $(N=$ Number, $E=$ Entrance face, $W=$ Width of fringes, and $S=$ Shear).

The first symbol, $N$, represents the number of sides or faces of the compound prism. When the numerical value of $N$ is significant its value will appear as a subscript ( $N_{4}$ for quadrilateral and $N_{6}$ for hexagonal prisms); otherwise $N$ appears without the subscript.

The second symbol, $E$, indicates the form of the entrance face of the prism. When $E$ appears without a subscript, the shape of the entrance face is not significant to the statement in which it appears. When the shape is significant it will be indicated by subscripts ( $E_{p}$ for plane and $E_{s}$ for spherical entrance face).

The third symbol, $W$, represents the maximum width fringes that can be obtained, with a perfect wave front, by moving the prism along the lens axis. The value of $W$ is finite if the direction of shear is parallel to the refracting edges of the prism (fig. 3) and the beam-dividing area is divided between the two component prisms (fig. 4). The value of $W$ is infinite if the direction of shear is perpendicular to the refracting edges of the prism, regardless of whether the beam-dividing areas are confined to one prism or divided between the components.

The lateral separation of the components of the principal ray, obtained by separating the planes of the two beam-dividing areas, corresponds to the introduction of a fixed wedge between the two component wave fronts. Similarily, the wedge between the wave fronts that is caused by a separation of the two coherent images of the source in the direction of shear may be called the variable wedge. When the directions of these wedges are the same they cannot be distinguished and the fringes act exactly as they do in a prism without a fixed wedge, When the direction of the fixed wedge is perpendicular to that of the variable wedge, the widths of the fringes in these two directions are proportional to the magnitude of the two wedges, respectively. The width in one direction (horizontally in fig. 2) is invariant and the width in the other direction (vertically in fig. 2) varies with the position of the prism relative to the lens (or point of convergence).

A prism with a built-in wedge that is perpendicular to the direction of shear will be designated by $W_{s}$ $(s$ stands for German senkrecht, meaning "perpendicular"). If the numerical value of $W$ is to be indicated also it will appear as a subscript of $W$ and expressed in units of milliradians. Thus a prism with a built-in wedge of $2.1 \mathrm{mrad}$ will be indicated by $N E W_{2.1 s} S$.

A prism with a built-in wedge that is parallel to the direction of shear will be designated by $W_{p}$ ( $p$ for "parallel"). Similarily, a prism with no built-in wedge (aluminum on one surface only) will be designated by $W_{\infty}$ because the magnitude of $W$ is given by this subscript.

It will be noted that the numerical value of $W$ in a $N E W_{p} S$-type prism is also infinity. However, it is desirable to discriminate between the three types and these symbols seem to be most appropriate.

The fourth symbol indicates the magnitude and direction of the shear. If the value and direction are insignificant to a statement in which it appears, the symbol $S$ will suffice. If shear is produced by a relative rotation of the prism component, to be described later, its direction will be parallel to the refracting edges of the prism and the corresponding symbol is $S_{p}$. If shear is produced by using prisms of different angles, the direction of shear will be perpendicular to the refracting edges of the prism and the corresponding symbol is $S_{s}$. When the numerical value of the shear is to be indicated it will appear in units of milliradians as a part of the subscript of $S$. Thus a shear of $2.1 \mathrm{mrad}$ in a prism having components of different angles will be indicated by $S_{2.1 s}$. Similarily, a prism having a shear of $2.1 \mathrm{mrad}$ that is introduced by a relative rotation of similar prism components will be designated by $S_{2.1 p}$. 


\section{Geometry of the Prism Interferometer}

The angles and size of the prisms for the shearing interferometer are not critical. The choice of size and angles of prisms that can be used to produce a workable instrument is so diverse that only optimum forms will be considered. These forms are chosen because they fulfill significant favorable operational performances that are unique for the different forms.

\subsection{Size of the Prism}

An optimum size prism (length of face $B$ in fig. 1) has a dividing plane of 1.5 in. A 1.0 in. or a 1.7 in. dividing plane would be almost equally satisfactory. Larger prisms require better glass and introduce larger aberrations because of thickness, unless a spherical entrance face is used. Small prisms are more difficult to polish and adjust than larger ones.

\subsection{Surfaces}

All reflecting surfaces of the prism interferometer are planes. The flatness of the beam-dividing plane is critical. The outer reflecting surfaces are less critical because the cones of light have small cross sections (being near the vertex of the cone) at the points of reflection. The entrance and exit faces may be planes if the angle of the cone of light does not exceed 1/8 rad (as when testing an $\mathrm{f} / 8$ lens with one conjugate relatively large). When larger cones are used, the spherical aberration of the prism introduces appreciable deformation of the wave front and consequently error into the results of measurement. This error is introduced at the entrance face because of the difference in refraction of any two rays that are combined to produce interference. Deformation of the wave front by the entrance face is practically eliminated if a planoconvex lens (figs. $5 \mathrm{E}$ and 5J) having the center of curvature of its spherical surface located near the point of internal reflection, $P_{1}$, is cemented to the entrance face.

Since any two rays that are combined to produce interference emerge parallel and nearly coincident, the shape of the exit face has little effect on the order of interference observed at any point in the aperture of the lens.

Distortion by a plane exit face does not contribute error to the measurements if they are made visually or photographically, provided reference points are established in the aperature of the objective being tested. If photography is used without previously established reference points (reference points inserted in the photograph), the distortion by the exit face will be appreciable for large cones of light but negligible for small cones.

\subsection{Angles of the Prism}

The angles of the prism are not critical. Since the magnitude of the angles does not affect the diffi- culty of construction, but does affect the performance, the choice of angles will be based on certain optimum constraints or requirements of performance. Thus, the angles will be so chosen that when the principal ray of light from a circular lens (circular cone of rays) enters the plane entrance face normally and at the center of this face (thus permitting a maximum size cone of rays to be accepted) the components of this principal ray will emerge from one exit face of the prism with minimum refraction. This condition provides minimum refraction for the entire cone of light and, consequently, minimum error in the results of measurement caused by aberration in the prism.

The prisms shown in figure 5 represent the several forms that are recommended. Other forms are possible but these are adequate and sufficient for all possible applications. For purposes of discussion the characteristic angles of the several prisms are represented as follows: the $N_{6} E W S_{p}$-type prisms by $\alpha^{\prime}, \beta^{\prime}$, and $\omega^{\prime}$ (fig. $5 \mathrm{~A}$ ); the $N_{4} E W S_{p}$-type prisms by $\alpha^{\prime}$ and $\beta^{\prime}$ (fig. $5 \mathrm{~F}$ ); the $N_{6} E W S_{s}$-type prisms by $\alpha_{1}, \alpha_{2}, \beta$, and $\omega$ (fig. 5B, the angles $\beta$ and $\omega$ may or may not be equal for the two component prisms); the $N_{4} E W S_{s}$-type prisms by $\alpha_{1}, \alpha_{2}$, and $\beta$ (fig. 5G). The angles shown here for all $N E W S_{p}$-type prisms represent the components of the prism angles in the plane of this drawing. Since the elements of these prisms are rotated (about an axis lying in this plane) to produce shear the refracting edges are no longer perpendicular to the figure. If the angle of rotation of any two prism components are $+\Omega$ and $-\Omega$, respectively, all refracting edges are at angles of $\left(90^{\circ} \pm \Omega\right)$ to the plane of figure 5 . The angles of the prisms shown here are $\alpha, \beta$, and $\omega$. The corresponding components of the prism angles in the plane of figure 5 are $\alpha^{\prime}, \beta^{\prime}$, and $\omega^{\prime}$. The relationship between any prism angle (unprimed) and its component in the plane of this drawing (primed) is

$$
\operatorname{Tan} X=\cos \Omega \tan X^{\prime},(X=\alpha, \beta \text {, or } \omega) .
$$

The deviation of the components of the principal ray out of the plane of figure 3 , at the points of internal reflection, $P_{1}$ and $P_{2}$, depends upon the angles $\alpha$ and $\Omega$. Since the deviations are equal and of opposite signs, and since the angle between the two emerging components from either exit face is the angle of shear, $\phi$, the approximate relationship between $\phi, \Omega$, and $\alpha$ is given by

$$
\tan 1 / 2 \phi=n \sin \Omega \tan \alpha,
$$

where $n$ is the refractive index of the glass.

The angle of shear in the $N E W S_{s}$ prism is, approximately,

$$
\phi=2 n\left(\alpha_{1}-\alpha_{2}\right) \text {. }
$$

When the angle $\alpha$, of the $N_{6} E W S_{p}$ prism is small and the components are rotated $\pi$ radians the prism becomes a $N_{6} E W S_{s}$ type. Such a prism, shown in 


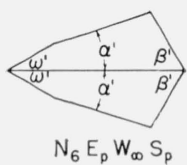

(A)

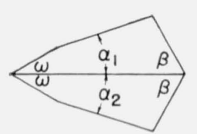

$N_{6} E_{p} W_{\infty} S_{s}$

(B)

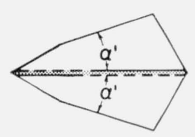

$N_{6} E_{p} W_{s} S_{p}$

(C)

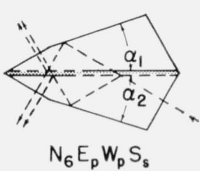

(D)

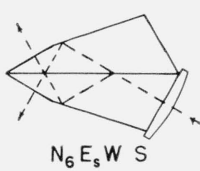

(E)

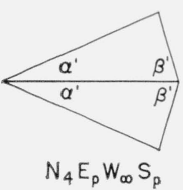

(F)

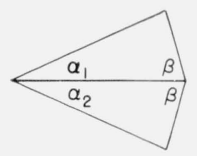

$N_{4} E_{p} W_{\infty} S_{s}$

(G)

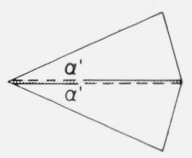

$\mathrm{N}_{4} E_{p} W_{s} S_{p}$

(H)

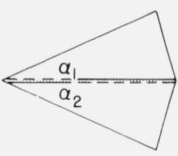

$N_{4} E_{p} W_{p} S_{s}$

(I)

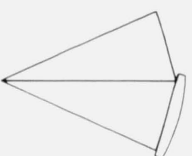

$N_{4} E_{s} W S$

(J)

Figure 5. Several different types of prism interferometers.

The letter-number abbreviations, described in section 3 , describe these prisms.

figure $6 \mathrm{~A}$ can be made with adequate shear and to accept an $f / 1$ cone of light. However, for testing such small $f$-value systems, spherical faces (fig. $6 \mathrm{~B}$ ) should be used on the prism to avoid spherical aberrations. When the angle of rotation, $\Omega$, equals $\pi$ radians, the angle, $\alpha$, of one prism is reversed and the shear, $\phi$, equals $4 n \alpha$. Consequently, the angle, $\alpha$, is usually small for such prisms.

Minimum refraction is obtained in plane face prisms when the principal ray of the entrance cone is normal to the plane entrance face and the two components of this principal ray, that emerge from each of the exit faces, make equal angles, but of opposite sign, with the exit face. If the prism is of the $N E W S_{p}$ type, the angles of emergence of the two pairs of beams are equal. However, the angles of emergence for the $N E W S_{s}$-type prisms, because $\alpha_{1} \neq \alpha_{2}$, cannot be a minimum for both pairs simultaneously. Consequently the pair of beams that emerges from face $A_{2}$ (fig. 1) will be ignored when considering the constraints of angles. When rays, $1^{\prime \prime}$ and $2^{\prime}$, (fig. 1A), that emerge from face $A_{1}$ suffer minimum refraction, their angles of emergence are $+(1 / 2) \phi$ and $-(1 / 2) \phi$, respectively, and the rays $1^{\prime}$ and $2^{\prime \prime}$, that emerge from face $A_{2}$ will be $0^{\circ}$ and $\phi$, respectively. The reason for minimizing refraction of the beams that emerge from face $A_{1}$, instead of those from face $A_{2}$, is due to their greater equality

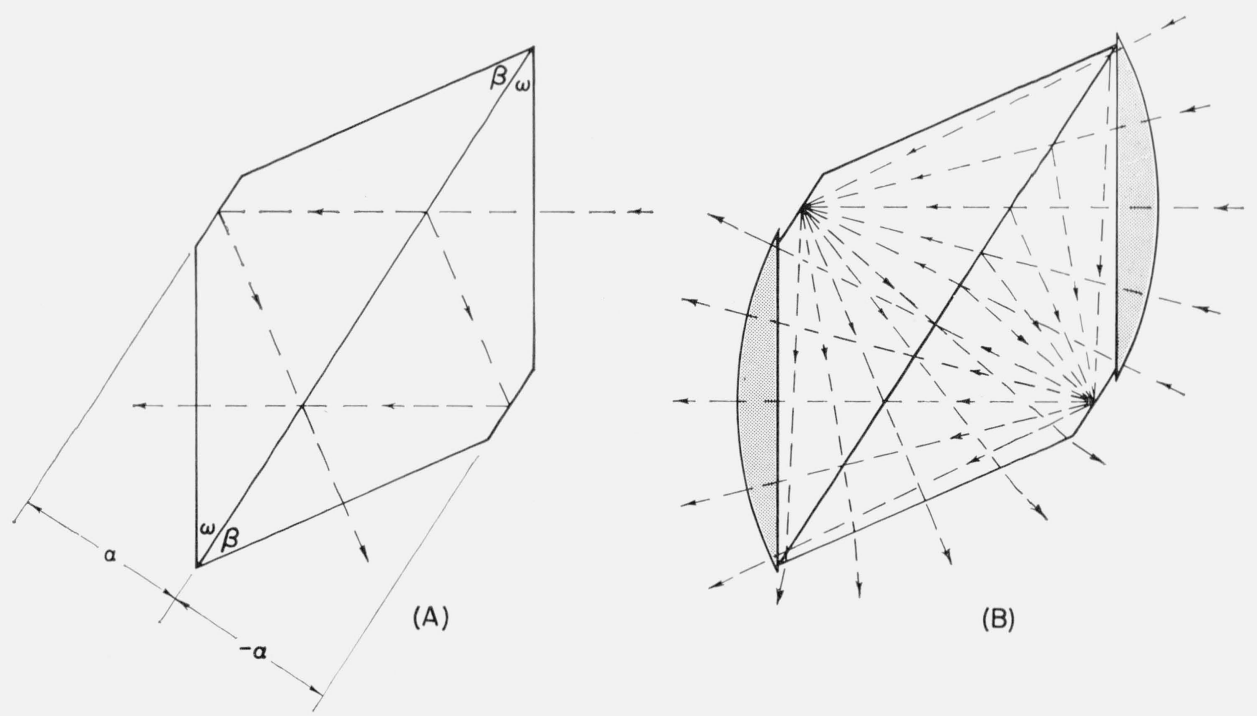

Figure 6A. Prism interferometer for testing very large cones of light.

B. Spherical entrance and exit faces eliminate error due to refraction of the prism surfaces. 
of intensity and, consequently, better visibility of fringes. This pair of beams will usually be used for making measurements.

It can be shown by simple triangulation that minimum refraction is obtained in plane face prisms if

$$
\left.\begin{array}{ll}
\beta=3 \alpha^{\prime} & \text { for all } N_{4} E_{p} W S_{p} \text {-type prisms, } \\
\beta=2 \alpha^{\prime}+\omega^{\prime} & \text { for all } N_{6} E_{p} W S_{p} \text {-type prisms, } \\
\beta=2 \alpha_{1}+\alpha_{2} & \text { for all } N E_{p} W S_{s} \text {-type prisms. }
\end{array}\right\}
$$

We will, therefore, impose these constraints on the design of all prisms described here. Since the face of prism $A_{1} B$ (fig. 1), that corresponds to the entrance face of prism $A_{2} B$, is not used it may be left unpolished. If, however, one wishes to be able to use either prism for receiving the light this surface should be polished and the angle $\beta_{1}$ should be equal to $\beta_{2}$.

The angles may have a variety of values consistent with eq (4). The range of these values depends upon factors such as the magnitude of the angle of shear (defined by $\phi$ ), the form of prism to be used, the refractive index of the glass, the maximum size cone of light that is to be used, and whether or not the outer reflecting surfaces are to be coated for increased reflection.

The range of usable values for $\alpha^{\prime}$ in the $N_{6} E W S_{p}$ prism is from $0^{\circ}$ to $\omega^{\prime}$. Obviously, as $\alpha^{\prime}$ approaches $\omega^{\prime}$ the $N_{6} E W S_{p}$ prism becomes $N_{4} E W S_{p}$ in form. The upper limit for $\alpha^{\prime}$ in the $N_{4} E W S_{p}$ prism is $\pi / 7$ rad $\left(=25.7^{\circ}\right.$, approximately). Figure 7 illustrates the limitations of $\alpha^{\prime}$ for this type of prism. If $\alpha^{\prime}$ $\left(=\omega^{\prime}\right)$ is greater than $\pi / 7 \mathrm{rad}$ (assuming the principal ray enters the center of the entrance face and normal

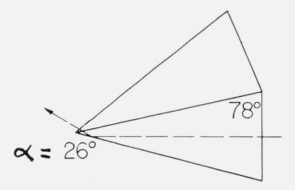

(A)

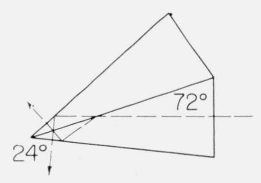

(C)

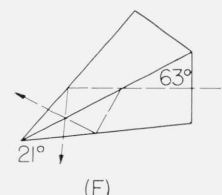

(F)

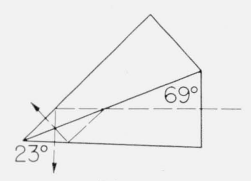

(D)

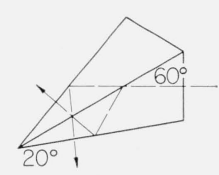

(G)

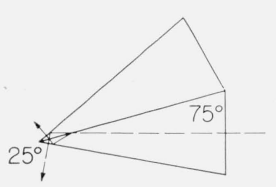

(B)
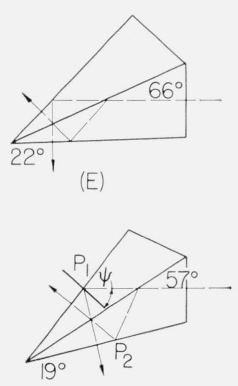

(H)
Figure 7. Several prism interferometers showing the effect on the rays, from above the upper limit of $\alpha\left(=26^{\circ}\right)$ to the lower practical limit $\left(=19^{\circ}\right)$. to it), the beam will not suffer two divisions at the beam dividing plane, as is shown in figure $7 \mathrm{~A}$.

The lower limit of $\omega^{\prime}\left(=\alpha^{\prime}\right)$ for the $N_{4} E W S_{p}$ prism depends upon whether the outer reflecting faces are coated to prevent transmission of light or, if not coated, on the refractive index of the glass. As shown in figure $7 \mathrm{H}$, the internal angle of incidence, $\psi$, increases with decreasing values of $\alpha^{\prime}$. Also the angle $\psi$ is different for different rays of the cone. When $\psi$ exceeds the critical angle of internal reflection, some of the light is transmitted at the internal reflecting surfaces and becomes lost. Consequently, if the principal ray is incident at the critical angle, then one-half of the observed image of the lens will appear poorly illuminated. If the reflecting areas of the prism do not appear in the emerging beam, these areas can be optically coated for increased reflection (as shown in fig. 8). The above discussion also applies to hexagonal prisms (fig. 8B). In this case, the entire areas of the reflecting surfaces may be coated.

A $N_{4} E W S_{p}$ prism having a value of $24^{\circ}$ for $\alpha^{\prime}$ will provide total internal reflection to a $15^{\circ}$ cone of light (corresponding to an $f / 3.8$ cone) if it is made of borosilicate crown glass $(n=1.517)$. The same shape prism, if made from a dense flint glass $(n=1.72)$, will provide total internal reflection to a $25^{\circ}$ cone, which corresponds to an $f / 2.3$ beam.

We have described in section 2 above how to obtain and control the angle of shear by rotating one prism relative to the other (all $N E W \dot{S}_{p}$ prisms), We have also described how shear may be obtained by using component prisms with different angles. Shear may also be obtained by purposely introducing (by grinding and polishing) pyramidal error into the prisms. Obviously this has the same effect on deviating the beams out of the plane of figure $1 \mathrm{~A}$ that a relative rotation of the components has when the two components are cut from a single original prism.

In general, when two surfaces are cemented together, as described above, they will not be parallel. Also the vertex of the wedge between them may have any direction. Consequently, neither component of this wedge, perpendicular or parallel to the edges of either prism component, will be zero.

If the dividing plane is confined to one surface, the cement effectively becomes a part of the component
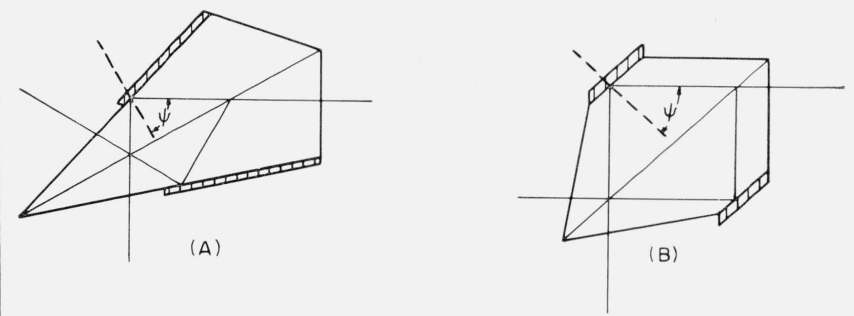

Figure 8. Prism interferometers with opaque reflecting surfaces to prevent transmission of light when the angle of incidence, $\psi$, is larger than the critical angle. 
prism that is uncoated and we have dissimilar prism components even though the original components were identical. In general, two of the angles are different and one prism has pyramidal error.

\section{Construction of the Prism Interferometer}

Only prisms of optimum form will be described. All prisms are assumed to be free from pyramidal error, i.e., all refracting edges of a prism component are assumed to be parallel.

\section{1. $\mathrm{N}_{6} \mathrm{E}_{\mathrm{p}} \mathrm{W}_{\infty} \mathrm{S}_{\mathrm{p}}$-Type Prisms}

Using the symbolic designation described in section 3 , the original WSI prism illustrated in figure $5 \mathrm{~A}$, was a hexagonal prism $\left(N_{6}\right)$, with a plane entrance face $\left(E_{p}\right)$. The two beam-dividing areas were produced by evaporating a semireflecting layer of aluminum on one of the inner surfaces of the prism ( $W_{\infty}$, dividing planes coplanar), and shear was introduced by a relative rotation of the two prism components.

Figure 9 illustrates how this prism was constructed from a single original prism (figure 9A) having angles $\beta, \gamma$, and $\omega$. None of these angles is critical but their values may be advantageously chosen, as described above. The angle $\gamma$ is removed by grinding and polishing a fourth face, $A$, that makes an angle, $\alpha$, between it and the opposite face, $B$, as shown in figure $9 \mathrm{~B}$. The prism $A B$ is then cut into two equal prisms, $A_{1} B_{1}$ and $A_{2} B_{2}$, by sawing it parallel to the plane of figure 9 This produces two quadrilateral prisms (figure 9C) that have identical angles, each of these component prisms being one-half as high as the original (minus the thickness of the saw cut).

A semireflecting coat of aluminum is put on surface $B_{2}$, and the two component prisms are cemented, together as shown in figure $9 \mathrm{D}$. The thickness and variations of thickness of the cement are not critical. Optimum conditions, however, favor a thin parallel film of cement that has optical properties approximating those of the glass prisms. The reflectivity of surface $B_{1}$ is negligible and the cement effectively becomes a part of prism $A_{1} B_{1}$.

\section{2. $\mathrm{N}_{6} \mathrm{E}_{p} \mathrm{~W}_{\infty} \mathrm{S}_{s}$-Type Prisms}

This prism is formed from two dissimilar prisms that have a difference in the two angles $\alpha_{1}$ and $\alpha_{2}$ (fig. 5B) that equals approximately one-half of the angle of shear divided by the index of refraction of the glass.

A practical procedure is to make two prisms, as described in section 5.1. above, with different values for $\alpha$. After sawing the prisms into equal parts the pairs are crossed by cementing one component of an original prism to a component of the other. Thus, two prisms of this type are obtained.

The reason for making prisms long enough to be sawed into two components is because two small surfaces are more difficult to polish than one larger surface.
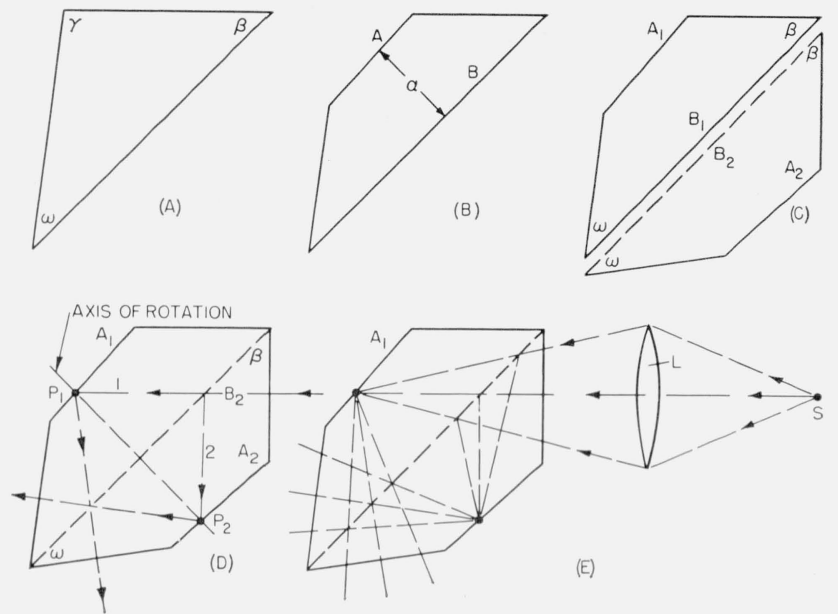

Figure 9. Several forms of a prism interferometer during stages of its construction and adjustment.

Figure $9 \mathrm{E}$ shows how the prism acts upon a converging beam of light to produce two coherent images of the source.

\section{3. $\mathrm{N}_{6} \mathrm{E}_{p} \mathrm{~W}_{s} \mathrm{~S}_{p}$ and $\mathrm{N}_{6} \mathrm{E}_{p} \mathrm{~W}_{p} \mathrm{~S}_{s}$-Type Prisms}

These prisms differ from the $N_{6} E_{p} W_{\infty} S$ (either $N_{6} E_{p} W_{\infty} S_{p}$ or $N_{6} E_{p} W_{\infty} S_{s}$ ), described above, by having semireflecting dividing planes on each of the two surfaces $B_{1}$ and $B_{2}$ of figure $9 \mathrm{C}$. The dividing plane of first incidence, $B_{2}$, extends from the vertex $\beta$ of prism $A_{2} B_{2}$ to a vertical line in this plane that passes through the normal projection of $P_{2}$ on to it. The dividing plane of second incidence, $B_{1}$, extends from the vertex $\omega$ of prism $A_{1} B_{1}$ to a vertical line in this plane that passes through the normal projection of $P_{1}$ onto it.

\section{4. $\mathrm{NE}_{s} \mathrm{WS}$-Type Prisms}

The only difference between this prism and the $N E_{p} W S$ described above is that a planoconvex lens is cemented to the entrance face. Best results are obtained if the lens and prisms are made of similar glass. The center of curvature of the convex surface should coincide approximately with the point $P_{1}$, where the principal ray suffers reflection from face $A_{1}$ of the prism.

\section{5. $\mathrm{N}_{4}$ EWS-Type Prisms}

The only difference between the $N_{4} E W S$ prisms and the corresponding $N_{6} E W S$ types described above is that the angle $\alpha$ is equal to $\omega$. This changes the component prisms from quadrilateral to triangular in form. They are easier to construct, easier to adjust, and in some respects, have significant advantages over the $N_{6} E W S$ types.

\section{The Beam-Dividing Plane}

The recommended optical coating for reflection and transmission of the beam-dividing plane is aluminum. Dielectric coatings that have been tried produced unfavorable polarization effects. 
The ratio of reflection to transmission is more critical in this prism interferometer than in many other types of interferometers. If we assume $R_{1}$ and $T_{1}$ to be the reflection and transmission, respectively, of the beam divider at the first incidence and $\dot{R}_{2}$ and $T_{2}$ the same at the second incidence, then the relative intensities of the several component beams may be designated by these quantities before and after each incidence on the beam divider. If we assume unit intensity for the beam before its first incidence, then the transmitted and reflected components are $T_{1}$ and $R_{1}$, respectively (see fig. 10). The $T_{1}$-beam is again divided at the second incidence into a $T_{1} T_{2}$-beam and a $T_{1} R_{2}$-beam. The $R_{1}$-beam is divided into a $R_{1} T_{1}$-beam and a $R_{1}$ $R_{2}$-beam.

If the beam divider is coated with a uniform film, the difference between $R_{1}$ and $R_{2}$ (or $T_{1}$ and $T_{2}$ ) is due only to the difference in angle of incidence. The difference in angle of incidence varies from one type prism to another with variation in the prism angles, and also from one ray to another in the converging beam.

If the first division of the beam occurs at the inner surface of one prism (see fig. 4) and the second division at the inner surface of the other prism, then the difference between $R_{1}$ and $R_{2}$ (or $T_{1}$ and $T_{2}$ ) may be decreased by placing one prism closer to the source during the aluminizing process.

The contrast in the fringe pattern depends upon the ratio of the intensities of the two beams that combine to produce interference. Since the diference between $R_{1}$ and $R_{2}$ (or $T_{1}$ and $T_{2}$ ) is usually much smaller than the difference $\left(T_{1}-R_{1}\right.$ or $\left.T_{2}-R_{2}\right)$, the pair of beams $R_{1} T_{2}$ and $T_{1} R_{2}$ are nearer equal than the pair $R_{1}$ $R_{2}$ and $T_{1} T_{2}$. Consequently, the former pair will produce the best contrast and are, therefore, the beams usually used for making observations. When the fringes are to be photographed the $R_{1} R_{2}-T_{1} T_{2}$ beams will be used for visual adjustments for and during exposure.

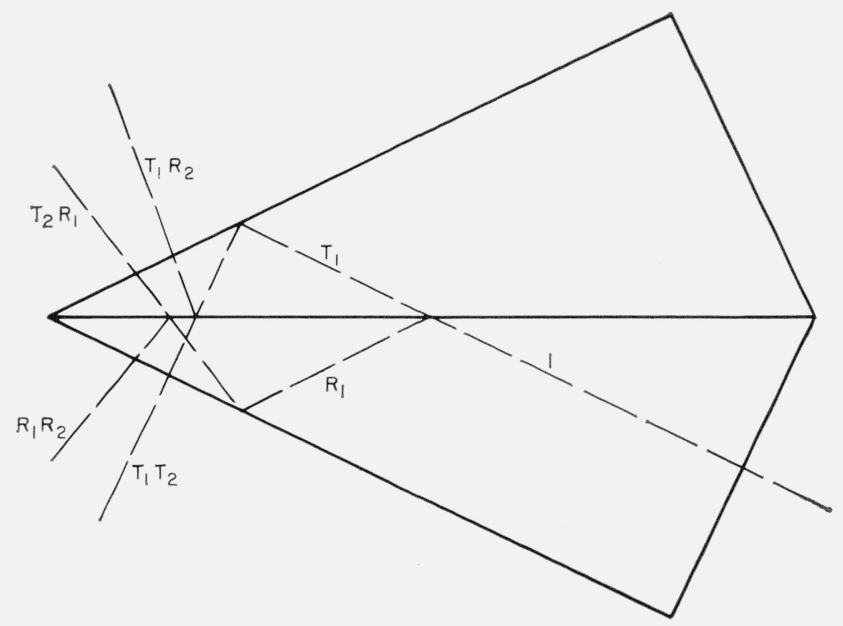

FIguRE 10. Ray trace showing relative intensities of the four emerging beams
An adequate test for the ratio of reflection to transmission of the beam divider is to observe visually the two images of a small source by means of the $R_{1} R_{2}-T_{1} T_{2}$ beams. If the images appear to differ in intensity by no more than a factor of about 2 , the contrast and intensity of fringes produced by the $R_{1} T_{2}-T_{1} R_{2}$ beams will be adequate.

\section{Optical Cement}

Any optical cement that can be worked as a liquid, becoming hard at ambient temperatures, can be used in these prisms. Canada balsam has been used quite successfully. Its hardness is adjusted, by distillation of volatile constituents, to a consistency considered proper by an experienced optician for cementing lenses.

The advantages of Canada balsam over other cements is that its viscosity may be varied at will with temperature, and almost unlimited time is available for making the adjustments. This cement should be cooked until it is quite hard at ambient temperatures. Other cements are more stable than Canada balsam, do not require elevated temperatures, and do not permit unwanted changes in the adjustments after the prism is put to use.

\section{Prisms Support and Ādjusting Screws}

The adjustment of the WSI prism is quite similar to that for the Kösters prism described in reference [8]. The two type prisms, $N_{6} E W S$ and $N_{4} E W S$, require different blocks for adjusting the prisms.

A photograph of the block used for adjusting: $N_{6} E W S$-type prisms is shown in figure 11. It consists of a compartment that approximates the shape and is slightly larger than the prism. Four vertical adjustable screws contact the bottom surface with

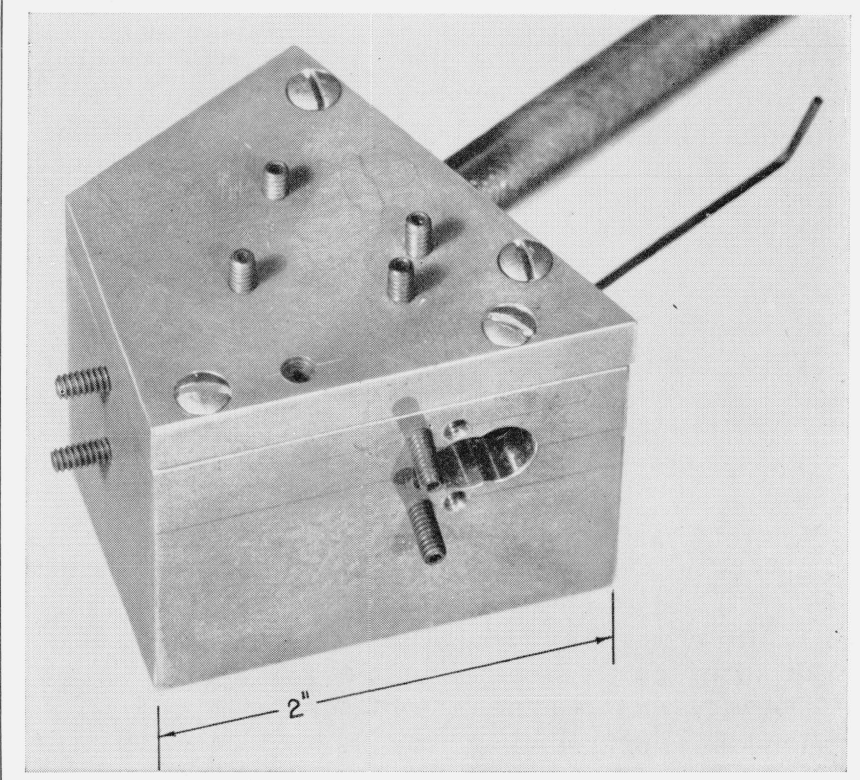

Figure 11. Photograph of adjustable block for adjusting $N_{6}$ EWS-type prisms. 


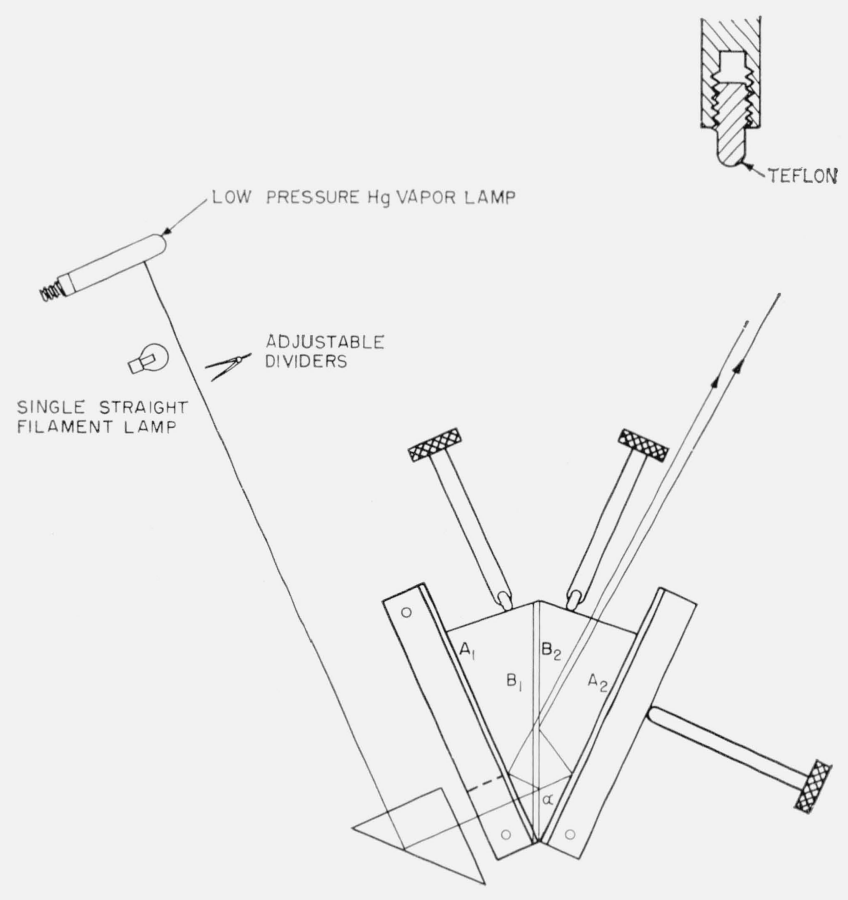

Figure 12. Schematic diagram of V-block for adjusting $\mathrm{N}_{4} \mathrm{EWS}$-type prisms.

The inset shows how the contacting ends of the screws are modified to avoid The inset shows how the contacting ends of the screws are modified to avoid
injuries to the glass surfaces. The adjustable dividers serve to measure the angle of shear.

two screws supporting each component prism. The top of the block has four vertical screws placed directly above those in the base of the block.

Two horizontal screws contact the entrance face of the prism and two others contact the exit face of the same component prism. Also, four similarly placed screws contact the corresponding faces of the other component prism. An entrance aperture, not shown in the photograph, and an exit aperture permit the light to pass through the prism to the observer. A supporting rod is shown for holding the block steady during the adjustment. This block may be heated on a simple hotplate.

The $V$-block for adjusting the $N_{4} E W S$ prisms illustrated in figure 12 is very similar to that used for the Kösters prism. Additional detail that applies to this adjustment appears in reference [8], which should be consulted.

\section{Illumination for Adjusting the Prism}

Monochromatic fringes of interference are obtained from a low-pressure mercury vapor lamp. This lamp is cylindrical in form, is approximately 3 $\mathrm{cm}$ in diameter, and $16 \mathrm{~cm}$ long. White light fringes are obtained from a galvanometer lamp that has a spherical globe $2.5 \mathrm{~cm}$ in diameter and a single straight filament. A right-angle prism is used to reflect the light into the prism.

The mercury lamp is located, as shown in figure 12 , approximately $120 \mathrm{~cm}$ from the prism. The galvanometer lamp is in line with the prism and mercury lamp and approximately $10 \mathrm{~cm}$ closer to the prism. This permits simultaneous use of both sources. The light passes through the prism in the reverse direction of that for normal use. This reversal of direction may be applied to almost any interferometer.

A low power telescope that permits a broad range of focusing is quite helpful for viewing and counting the interference fringes that are formed in the prism. These fringes are localized as in the conventional Mach-Zehnder interferometer.

\section{Adjustment of the Prism Interferometer}

The adjustment of quadrilateral prisms $\left(N_{4} E W S\right)$ is no different from that of hexagonal prisms $\left(N_{6} E W S\right)$. Consequently, a description of one type is sufficient. Since the former type is simpler in structure and easier to adjust because of differences in the mechanism for performing the adjustments, the $N_{4} E W S$ type will be used for this description.

If the entrance face is to be spherical $\left(N E_{s} W S\right)$, a plane entrance face prism is constructed and adjusted before the planoconvex lens is cemented to the plane entrance face.

A description of the adjustments of all types of prisms is provided by describing those for the four types shown in figure 13. Figures 13A and 13B show prisms with dissimilar components $\left(\alpha_{1} \neq \alpha_{2}\right)$. Figures $13 \mathrm{C}$ and $13 \mathrm{D}$ show prisms with similar components. The two beam divisions occur at a common dividing plane in figures $13 \mathrm{~A}$ and $13 \mathrm{C}$ and at different planes in figures 13B and 13D

There are four adjustments to be performed on a prism. Some of these are important and some are relatively unimportant to the performance of the prism. These adjustments are as follows: (1) to adjust the optical path of the two components of the
(A)

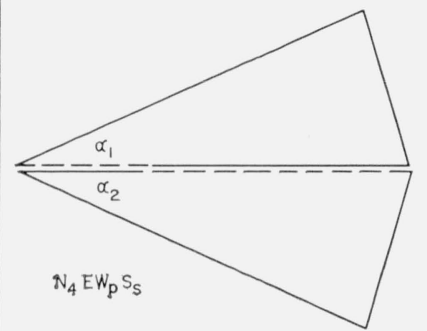

(B)

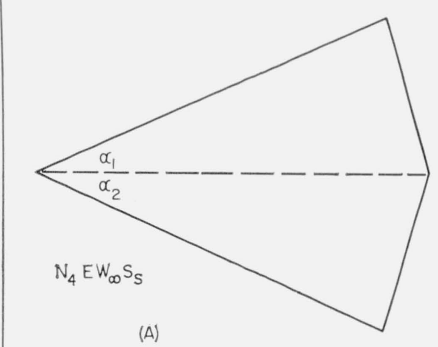

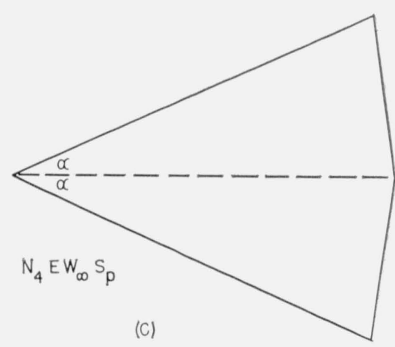

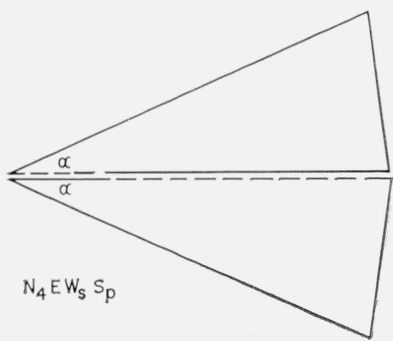

(D)
Figure 13. Four types of prism interferometers. 
principal ray 1 and 2 (fig. 9D) to equality so that the zero order of interference is centered in the fringe pattern; (2) to adjust the thickness, $t$, of the cement; (3) to parallel the film of cement so that surfaces $B_{1}$ and $B_{2}$ (fig. $9 \mathrm{C}$ ) are approximately parallel to each other; and (4) to adjust the magnitude of the angle of shear, $\phi$.

There is no definite order for making these four adjustments except that number (4) should be fourth and last. The other three can be performed in any order. However, by first making adjustment number (1), and maintaining it, fringes of good contrast are available for making the other adjustments.

\subsection{Interferometric Fringes for Observing Prism Adjustments}

There are two types of interference fringes that are used for making prism adjustments. One of these sets of fringes is nonlocalized and the other is localized with a high degree of variability in the position of the plane of localization. The nonlocalized fringes are produced by two images of a small source. A lens or mirror must be used to produce an image of the source and the prism forms two images of it by amplitude division of the beam. The arrangement shown in figures 1 and 9 with a suitable source will fulfill the requirements. The direction and angular width of the nonlocalized fringes, mentioned in section 2 above, depend, respectively, upon the direction of the line joining the two coherent images of the source and on the angle subtended by this line at the point where the fringes are observed. This source must be small [1] in the direction of shear. The nonlocalized set of fringes is unnecessary for making adjustments except when a large angle of shear is used. When $\phi$ is large the localized fringes cannot be seen without the use of a long-focus microscope because they are so closely spaced.

The localized fringes may be used for making all necessary adjustments if the value of $\phi$ is not too large (approximately 0.02 rad). These fringes are, in all respects, similar to the fringes produced by a Mach-Zehnder [6] interferometer. They may be localized anywhere from $+\infty$ to $-\infty$. The plane of localization shifts so much during initial adjustments that a low-power telescope with a large focusing range is quite useful. These fringes may also be seen with the unaided eye. An extended source may be used when the fringes have moderate spacing and the order is small, but a source that is narrow in the direction of shear improves the contrast and is necessary when the fringes are very closely spaced.

When the dividing plane is confined to one plane as in the $N E W_{\infty} S$-type prisms (figs. $13 \mathrm{~A}$ and $13 \mathrm{C}$ ) and an extended or narrow source is used without a lens, as shown in figure 12 , only one set of fringes is seen. When the dividing planes are divided between the two prism components (figs. 13B and $13 \mathrm{D})$, three sets of fringes are seen. Figure 14 illustrates how the paths of the light rays differ for the three sets.

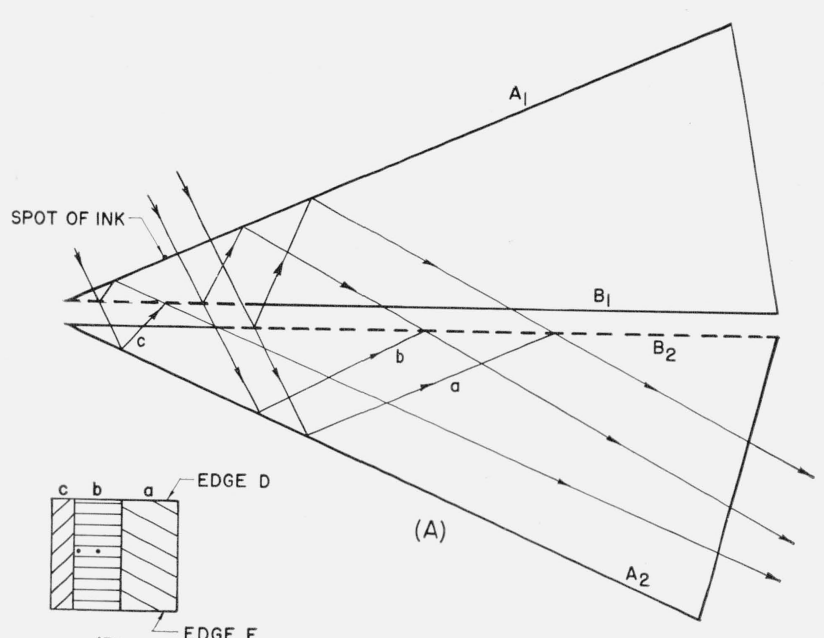

(B)

FIGURE 14A. Ray trace showing how three sets of interference fringes are obtained when the dividing planes are on the two surfaces $B_{1}$ and $B_{2}$.

B. Appearance of fringes when illuminated as illustrated in figure 12. The edges, $D$ and $E$, are the intersections of face $A_{2}$ with the base and top surfaces of the prism.

\subsection{Preparation of Prism for Adjustments}

The prism components are cemented together initially in much the same manner as described for the Kösters [8] prism. The cement is assumed to be Canada balsam. The faces of the prism are cleaned. A small spot of ink is then placed on the exit face, $A$ (which becomes the entrance face during the adjustment), approximately $2 \mathrm{~mm}$ from the vertex of angle $\alpha$ and midway between the edges $D$ and $E$ (fig. 14B) of the prism. The compound prism is placed in the adjustable $V$-block (fig. 12 ) and the assembly in an oven. See reference [8] for oven characteristics. Using the mercury lamp, illustrated in figure 12, and observing the spot of ink one sees three spots. One is a direct image of the inkspot and the other two are images in silhouette produced by light reflected from faces $A_{1}$ and $A_{2}$. The images of this spot aid the observer in finding fringes and in placement of the zero-order fringe when adjustments are complete.

\subsection{Adjustment Procedure}

The separation of the two images of the spot of ink, described above, is a coarse (or rough) measure of the prism adjustment. A low-power telescope or a long-focus microscope is convenient for observing both the spot and the interference fringes. A lateral separation (in the direction perpendicular to edges $D$ and $E$, fig. 14B) of the images indicates either a relative rotation of the prism components or a lateral wedge in the cement. A separation in the direction perpendicular to the vertex of $\alpha$ indicates a difference in the two light paths. In general, if one prism is moved relative to the other in this latter direction, the two images change their relative separation in this direction and, consequently, can 
be made to approach coincidence. When they're close together, fringes of interference can usually be seen in the section (a, b, or c of fig. 14B) in which the images are seen.

If the prism is of the $N E W_{p} S_{s}$ or $N E W_{s} S_{p}$ type, the original inkspot should be located at a distance from the vertex, $\alpha$, such that the two images of it appear in the central section, b, of figure $14 \mathrm{~B}$, because this section transmits the light when the prism is used later for making measurements. The relative movement of the images of the inkspot is performed by sliding one prism on the other with the cement as a fluid lubricant. Heating the cement permits the viscosity to be altered at will.

After interference fringes are obtained with the mercury lamp their visibility can usually be improved by reducing the order of interference. When the order approaches zero, white light fringes may be seen with the tungsten lamp. Both lamps may be in use simultaneously. The order of interference is altered by a relative movement of the prisms perpendicular to their refracting edges (vertices of the angles).

When the direction of the wedge is known the adjustable $V$-block and its screws can be adjusted to correct the cement wedge. Reducing the angle of the block and applying pressure to the tops of the prisms causes the cement wedge to become thinner at the top. Conversely, by opening the $V$-block wedge until it is larger than the sum of the prism angles, $2 \alpha$ or $\left(\alpha_{1}+\alpha_{2}\right)$, the cement wedge becomes thinner at the bottom. These adjustments are described in reference [8] and apply equally well to this prism. A zero wedge in the cement is desirable for all prism types $N E W S_{p}$.

The wedge in the cement of prism types $N E W_{p} S_{s}$ (fig. 13B) is unimportant. This prism is relatively easy to adjust if the angle $\left(\alpha_{2}-\alpha_{1}\right)$ is not large. The fringes are rotated parallel to the refracting edges of the prism and the zero order is adjusted to center (on the inkspot shown in fig. 14A). Best compensation is obtained for white light use if the thickness of the cement is small. The effect of cement thickness on the nature of the interference fringes is insignificant in prism types $N E W S_{s}$ (fig. 13B) but is important in prism types $N E W_{s} S_{p}$ (fig. 13D).

The localized fringes in prism types $N_{4} E W_{\infty} S_{3}$ (fig. 13A) will always be relatively narrow, since the angle between the two emerging wave fronts approximates the angle of shear and is usually too large to yield broad fringes. The wedge in the cement adds to this angle and is difficult to distinguish from the component due to the prism angles. However, the cement wedge can always be reduced to insignificance by heating it to produce low viscosity and applying a little pressure on the prisms. Even if a wedge remains in the cement of this prism, it can be counteracted or compensated for by rotating one prism relative to the other until the fringes are parallel to the vertices of the prism angles. Thus, a residual wedge in this prism does little harm.

The localized fringes in the $N_{4} E W_{\infty} S_{p}$-type prism (fig. 13C) will be very broad if there is no wedge in the cement and no relative rotation of the component prisms. If the fringes become parallel to the prism's refracting edges when one prism is rotated about an axis normal to the dividing plane, the vertex of the cement wedge is parallel to that of the prism. The maximum fringe width, during this rotation of prism components, is then a measure of the magnitude of the wedge. The direction can be determined by the usual test for increasing orders. If the reflecting film is on surface $B_{2}$ (fig. 12) and the cement is thicker at the top than at the bottom, the fringes will move away from the vertex of angles $\alpha$ when prism $A_{2} B_{2}$ is moved downward. Also, they will move toward the vertex of $\alpha$ when prism $A_{1} B_{1}$ is moved downward or prism $A_{2} B_{2}$ is moved upward.

The light that passes from a source, arranged as indicated in figure 12, through a $N E W_{s} S_{p}$-type prism produces three sets of interference fringes. These are indicated in figure $14 \mathrm{~B}$ and labeled $a, b$, and $c$. One of the two interfering beams in each set is reflected from $A_{1}$. The second beam in each set travels the following paths: beam $a$ is reflected from $B_{2}$ to $A_{2}$ and finally from $B_{2}$ again; beam $b$ is reflected from $B_{2}$ to $A_{2}$ and finally from $B_{1}$; beam $c$ is reflected from $B_{1}$ to $A_{2}$ and finally from $B_{1}$ again. The set $a$ is identical to the fringes seen in prism type $N E W S_{p}$ (fig. 13C). Set $c$ is the same as would be seen in this prism should it be rotated $180^{\circ}$ in the adjustable $V$-block. The fringes in set $b$ are independent of the wedge in the cement (assuming plane surfaces and homogeneous media). Consequently, these fringes will always be normal to the refracting edges of the prism. A relative rotation of the prisms will cause these fringes to become very broad. This occurs when $\Omega$ (defined above) becomes equal to 0 . When this condition exists the fringes in set $a$ are parallel to the vertex of the wedge formed by the two surfaces $B_{1}$ and $B_{2}$. Also, the width of these fringes is a measure of the magnitude of this wedge. The direction of increasing thickness of the cement wedge is the same as the direction of movement of the fringes (in set $a$ ) when prism $A_{2} B_{2}$ (fig. 12) is moved downward. When the direction and magnitude of the cement wedge are known, corrective measures may be taken to reduce it. When the surfaces $B_{2}$ and $B_{1}$ become parallel, all three sets of fringes will remain equally wide as $\Omega$ is varied for introducing shear. The angle of relative rotation of the prisms, $\Omega$, may be held to a value that produces fringes of a convenient width for counting. By adjusting the zero-order fringe in set $b$ to a reference point on the boundary between $b$ and $c$ the difference in order between sets $b$ and $c$ can be counted while component prism $A_{1} B_{1}$ is moved upward relative to component $A_{2} B_{2}$. This difference in order multiplied by the wavelength of the light is equal to $2 t \sqrt{n_{2}^{2}-n_{1}^{2} \sin ^{2} 3 \alpha}$, where $t$ is the thickness of the cement at the bottom of the prism (fig. 12), $n_{2}$ and $n_{1}$ are the refractive indices of the cement and glass, respectively. Thus the thickness of the cement wedge may be measured.

A similar count of the difference in order between sets $a$ and $b$ will also give a value for $t$. The change 
of zero order in set $b$ to zero order in set $a$ is obtained by moving component prism $A_{2} B_{2}$ downward relative to component $A_{1} B_{1}$. This difference in order multiplied by the wavelength equals $2 t \sqrt{n_{2}^{2}-n_{1}^{2} \sin ^{2} \alpha}$. Since this latter quantity is larger than that for sets $b$ and $c$ by a factor of 2 or 3 , and since this count is not critical, the smaller value will usually be used for obtaining $t$.

If $t$ becomes too small it can only be increased by recementing the prisms. Spacers in the form of paper strips placed along the margins (edges $D$ and $E$, fig. $14 \mathrm{~B}$ ) will serve to control the thickness of the cement. If $t$ is found to be too large, it can be readily reduced by prolonged pressure on the prisms which causes the cement to be forced from between the prism components. Usually a value of $t$ that is within 50 percent of the optimum value will be satisfactory.

When the required value of $t$ is obtained the prism components are rotated to produce the desired shear, the zero-order fringe is adjusted to the center of the prism aperture, and the temperature is reduced sufficiently slowly (approximately $25^{\circ}$ per hour or less) to avoid excessive gradients during cooling.

\section{Angular Deviation by the Prism}

The mean deviation of a pair of interfering beams is the angle between the entrance beam and the bisector of the angle between any two emerging beams that combine to produce interference.

The range of deviation of interfering beams that may be obtained with this prism interferometer is considerable. There may be occasions when it is desirable to have a mean deviation of a pair of interfering beams equal to $0^{\circ}, 90^{\circ}$, or both. The $N_{4} E W S$ prism cannot be made to produce zero deviation. A $90^{\circ}$ mean deviation, however, is obtained with the $N_{4} E W S_{p}$ prism by making $\alpha=22.5^{\circ}$. The $N_{6} E W S_{p}$ prism will produce a $90^{\circ}$ mean deviation of two interfering beams if $(\omega+\alpha)=(\beta-\alpha)=45^{\circ}$. The $N_{6} E W S_{s}$ prism will produce a mean deviation of $0^{\circ}$ for one pair of beams and $90^{\circ}$ for the other pair of interfering beams if $\alpha_{1}=-\alpha_{2}$ and $\beta=\omega=45+2 \alpha_{1}$.

\section{Summary}

Many types of prisms have been constructed and tested. They are found to be quite useful for measuring the shapes of wave fronts and changes in wave fronts with time. Prisms with large shear angles yield higher accuracy when testing high-quality (telescopic) optical systems, and prisms with small shear angles yield relatively high accuracy when testing systems that have large aberrations (large OPD variations).

Important advantages of the several prism types are: $N_{6} E W S$ prisms can be most economically made by grinding and polishing one surface, $A$, (fig. 9B) on right-angle reflecting prisms, which are readily available commercially. $N_{4} E W S$ prisms are easier to make from unworked glass because only three faces have to be polished, instead of the four required for the $\mathrm{N}_{6} E W S$ prisms. The range of deviations for the $N_{6} E W S$ prisms is different from that of the $N_{4} E W S$ type, and this is sometimes important. The vertex of the cone of light, received by an $N_{4} E W S$ prism (image $S$, fig. 1), can be much closer to the exit face than for the $N_{6} E W S$ prism, which is important for observation of large cones of light from high-power optics, because the size of the pupil is limited. The $N E W_{s} S$ prism permits rotation of the fringes whereas the $N E W_{p} S$ prisms do not. The $N_{6} E W S$ prism can be made to accept a larger cone of light than that for the $N_{4} E W S$ type. The $N_{4} E W S$ prisms requires a simpler fixture for making adjustments (fig. 12) than does the $N_{6} E W S$ type (fig. 11). These and other differences permit the choice of a best type for any given application.

All prisms used in this development were made by Mr. John V. McDermott.

\section{Appendix A}

\section{Reference Points}

The analysis of data taken with the WSI requires that the reference points be chosen in a manner that permits the application of the operations described in reference [5]. The reference points should be equally spaced on a straight line (usually a diameter of the lens or mirror being tested) that is parallel to the direction of shear. The angular spacing of the points is equal to the angle of shear. Such a set of points is defined as a "family" of reference points.

If the reference points are labeled (see fig. 15A) $P_{0}, P_{1}, P_{2} \ldots P_{N}$, where $(N+1)$ is the number of points in the family, the corresponding images will be labeled (fig. 15B) $P_{0}^{\prime}$ and $P_{0}^{\prime \prime}, P_{1}^{\prime}$ and $P_{1}^{\prime \prime}, P_{2}^{\prime}$ and $P_{2}^{\prime \prime}, \ldots P_{N}^{\prime}$ and $P_{N}^{\prime \prime}$. One image of each point will coincide with images of the adjacent points. One image of $P_{2}$ (i.e. $P_{2}^{\prime}$ ) will coincide with image $P_{1}^{\prime \prime}$ and the other image of $P_{2}$ (i.e. $P_{2}{ }^{\prime \prime}$ ) will coincide with image $P_{3}{ }^{\prime \prime}$. If one point (such as $P_{2}$ in fig. $15 \mathrm{C}$ ) is displaced from its family position the images of it will not concide with those of the neighboring points (fig. 15D). If the line of reference points (fig. 15E) is not parallel to the direction of shear the images will not coincide in pairs.

The number of points in a family depends upon the angle of shear and the angular aperture of the lens or mirror being tested. The ratio of aperture angle to shear angle is equal to the number of spaces between the points plus the excess spaces between the border and end points. The excess fraction of spaces allows for a possible change of one in the number of members of a family. For instance, if there are $3 \frac{1}{2}$ spaces and one point is chosen at the center, then only 3 points can be included in the family. However, if one point is chosen near the margin, there will be 4 points in the family.

The position of the family of points on the line is not critical. When one point is chosen, however, the positions of all members of the family are known. 
The chosen point may be at the center, at the margin, at any chosen distance from the margin or center, and on any line parallel to the direction of shear. The chosen line is usually a diameter of the circular aperture (if it is circular) but may be any chord of this circle that is parallel to the direction of shear and of sufficient length.

It sometimes happens that the number of points in a family is too small and, consequently, the spacings are too large for sufficient data. For example, if an $f / 11\left(5.2^{\circ}\right.$ aperture angle) telescope objective is to be tested with a prism whose angle of shear equals one degree (see fig. 16), the largest number of reference points that may be included in a family is six. This is obtained by choosing one point (whose images, $P_{0}^{\prime}$ and $P_{0}^{\prime \prime}$, appear as dots in the figure) near the margin. If one point (whose images $P_{5}^{\prime}$ and $P_{5}^{\prime \prime}$ appear as small circles in fig. 16) is chosen at the center of the lens, it will be found that the family consists of only five points; and since the number of reference points in the fringe pattern is one less than the number in the family, there will only be four points for reading data. Five or six points may not provide data at sufficiently close intervals along the line of reference points - especially if the optics that are being tested have zonal errors.

The number of usable points along the line of reference points may be increased by using two or more families. Thus, two families of points (labeled family No. 1 and family No. 2, in fig. 16) may be combined to decrease the angular spacing to $0.5^{\circ}$. A third family may be added to provide additional points. Any integral number of families may be added to obtain data at as many points as desired.

Reference [5] describes how to compute for one family of points. We will show here how to apply more than one family. When two or more families of points are combined, it is desirable to use a different unit for $v$ and to choose equal spacings for the points. This permits the use of integers for subscripts with no added complications. When two families are to be used, the separations of the points equal one-half the shear. When three families are used, the spacing is one-third the shear, etc.

We will use the fringe pattern of reference [5] (shown in fig. 17A) to show how to apply two families of points. The second family is added (fig. 17B) as small circles located midway between adjacent points of the original family of points. The orders of interference, for all points in the fringe pattern, are listed in column 2 of table 1.

The equations of observation (No. 5, ref. [5]) for family No. 1 are

$$
\begin{aligned}
& \delta_{2}=\delta_{0}+2.74+r-2 \epsilon \\
& \delta_{4}=\delta_{2}+2.10+r-4 \epsilon \\
& \delta_{6}=\delta_{4}+1.70+r-6 \epsilon \\
& \delta_{8}=\delta_{6}+1.45+r-8 \epsilon \\
& \delta_{10}=\delta_{8}+1.30+r-10 \epsilon \\
& \delta_{12}=\delta_{10}+1.00+r-12 \epsilon \\
& \delta_{14}=\delta_{12}+0.30+r-14 \epsilon
\end{aligned}
$$

and the set for family No. 2 are
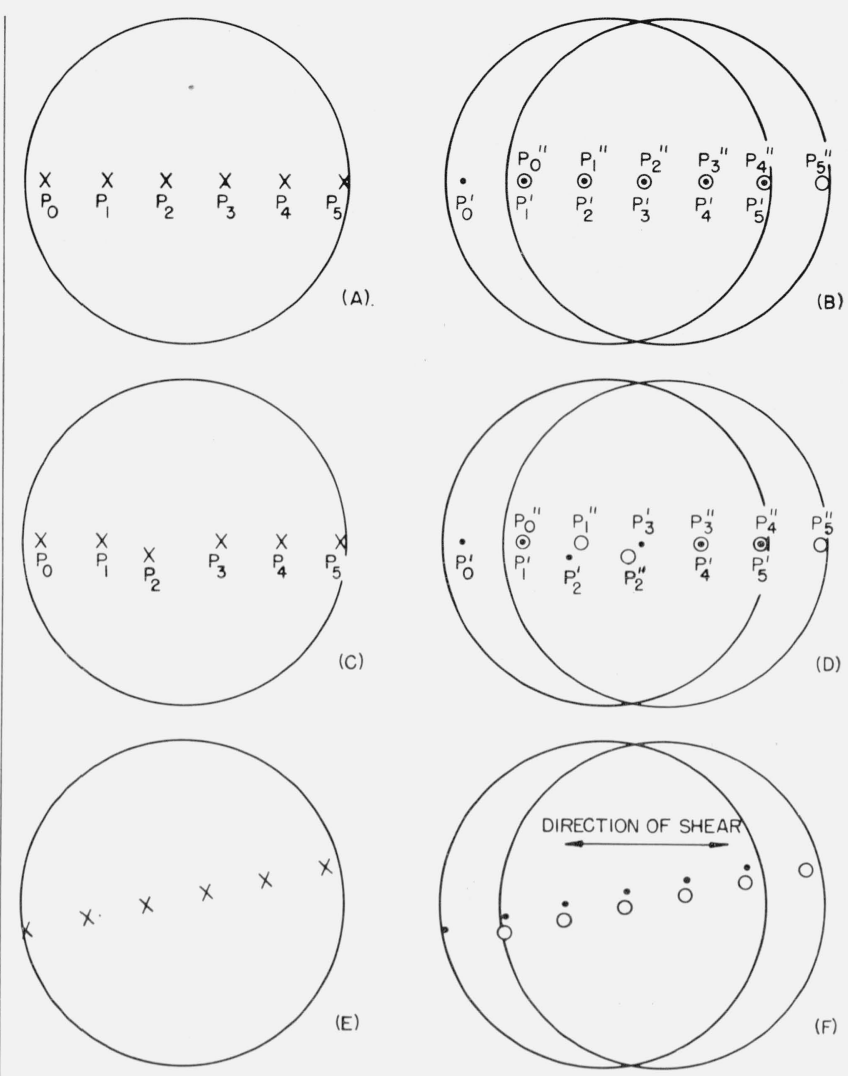

Figure 15. Illustration of the characteristics of a family of reference points.

$$
\begin{gathered}
\delta_{3}=\delta_{1}+2.40+r-3 \epsilon \\
\delta_{5}=\delta_{3}+1.88+r-5 \epsilon \\
\delta_{7}=\delta_{5}+1.56+r-7 \epsilon \\
\delta_{9}=\delta_{7}+1.37+r-9 \epsilon \\
\delta_{11}=\delta_{9}+1.18+r-11 \epsilon \\
\delta_{13}=\delta_{11}+0.71+r-13 \epsilon
\end{gathered}
$$

When using two or more families of reference points, the methods of solution described in reference [5] are impractical. The deviations ( $\delta$ 's) of one family will not, in general, fall on the same curve (which represents the shape of the wave front) that passes through the other family of deviations. The two families may be required to fall on one and the same wave front shaped curve by applying Newton's interpolation formula [9] and requiring that it be a polynomial of degree $(N-1)$. This is obtained by requiring that,

$$
\begin{aligned}
\delta_{0}+ & (1-N) \delta_{1} \\
& +\frac{(1-N)(2-N) \delta_{2}}{2 !}+\frac{(1-N)(2-N)(3-N) \delta_{3}}{3 !} \\
& +\cdots+\frac{(1-N)(2-N) \ldots(-1) \delta_{(\mathrm{N}-1)}}{(N-1) !}=0
\end{aligned}
$$

where $N$ is the total number of points in the two families. 


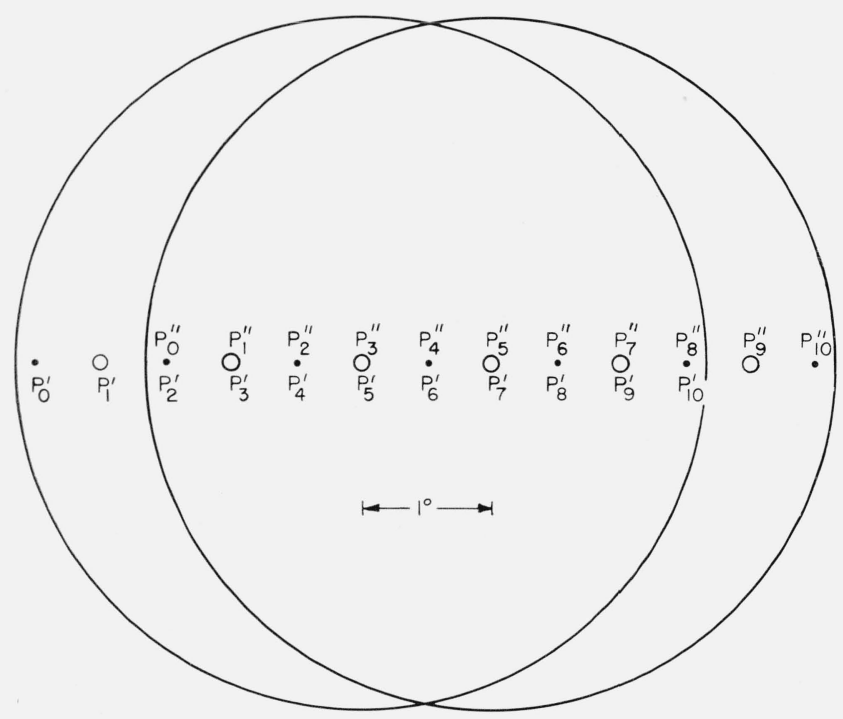

- - - family (OF REFEREnCe points) no.I

O O FAMILY (OF REFERENCE POINTS) NO.2

FIgURE 16. Illustration of how two families of points may be combined to increase detail in the computed wavefront.

The dots, labeled $\mathrm{P}_{0}, \mathrm{P}_{2}, \mathrm{P}_{4}, \mathrm{P}_{6}, \mathrm{P}_{8}$ and $\mathrm{P}_{10}$ form a family of points that are defined as family No. 1. The circles, labeled $\mathrm{P}_{1}, \mathrm{P}_{3}, \mathrm{P}_{5}, \mathrm{P}_{7}$, and $\mathrm{P}_{9}$ form another family of points that are defined as family No. 2.

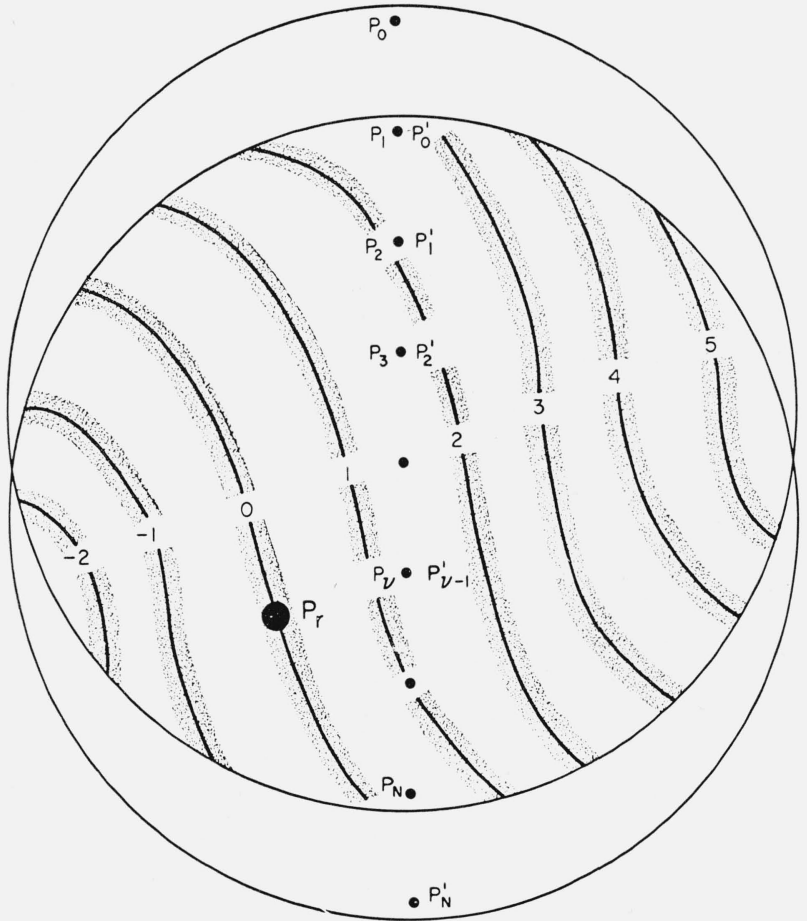

A
The two families of deviations are referred to a common reference sphere by requiring that

$$
\left.\begin{array}{l}
\delta_{0}+\delta_{1}+\delta_{2}+\delta_{3}+\delta_{4}=0 \\
\delta_{5}+\delta_{6}+\delta_{7}+\delta_{8}+\delta_{9}=0 \\
\delta_{10}+\delta_{11}+\delta_{12}+\delta_{13}+\delta_{14}=0
\end{array}\right\}
$$

and the deviations are required to fit the same wave front curve by requiring ( $N=15$ in equation (12)) that

$$
\delta_{0}-14 \delta_{1}+91 \delta_{2}-\ldots \delta_{14}=0
$$

On combining eqs (10), (11), (13), and (14) and solving them as a set of simultaneous equations, we obtain the deviations shown in table 1, column 3 .

Equation (14) may be replaced by the equivalent simpler equation,

$$
\begin{aligned}
15\left(\delta_{1}+\delta_{13}\right)-90\left(\delta_{2}+\delta_{12}\right)+ & 365\left(\delta_{3}+\delta_{11}\right) \\
-1000\left(\delta_{4}+\delta_{10}\right) & +2003\left(\delta_{5}+\delta_{9}\right) \\
& +3002\left(\delta_{6}+\delta_{8}\right)-3432 \delta_{7}=0,
\end{aligned}
$$

which is obtained by subtracting $\sum_{\nu=\alpha}^{14} \delta_{2}$ (which from 13 , equals 0 ) from 14.

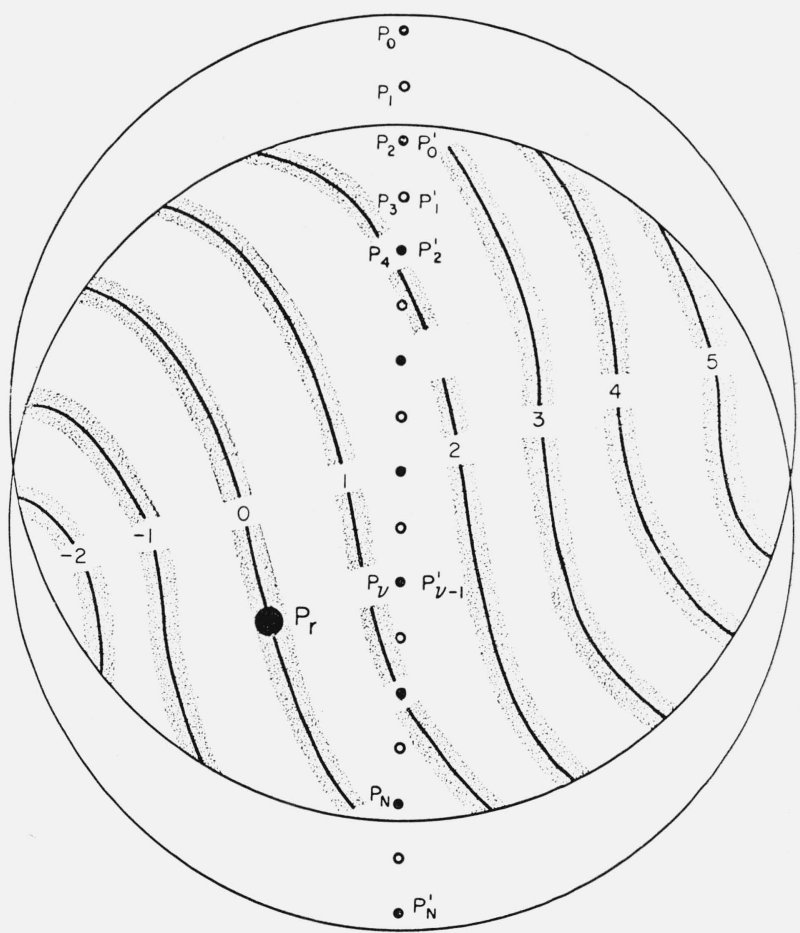

$\mathbf{B}$

Figure 17A. A fringe pattern with one family of points.

B. A fringe pattern with two families of points. 
'TABLE 1. Observed orders of interference, $q_{\nu}$, and computed deviations, $\delta_{\nu}$, from figure 17

\begin{tabular}{|c|c|c|}
\hline$\nu$ & $q_{v}$ & $\delta_{\nu}$ \\
\hline $\begin{array}{l}0 \\
1 \\
2 \\
3 \\
4\end{array}$ & $\begin{array}{l}-. \\
2.74 \\
2.40 \\
2.10\end{array}$ & $\begin{array}{r}-0.28 \\
-.05 \\
.09 \\
.13 \\
.11\end{array}$ \\
\hline $\begin{array}{l}5 \\
6 \\
7 \\
8 \\
9\end{array}$ & $\begin{array}{l}1.88 \\
1.70 \\
1.56 \\
1.45 \\
1.37\end{array}$ & $\begin{array}{r}.07 \\
.02 \\
-.02 \\
-.04 \\
-.02\end{array}$ \\
\hline $\begin{array}{l}10 \\
11 \\
12 \\
13 \\
14\end{array}$ & $\begin{array}{r}1.30 \\
1.18 \\
1.00 \\
0.71 \\
.30\end{array}$ & $\begin{array}{r}.04 \\
.09 \\
.11 \\
.01 \\
-.24\end{array}$ \\
\hline
\end{tabular}

(Paper 68C3-160)

\section{References}

[1] Bates, W. J., Proc. Phys. Soc. (London), 59, 940 (1947).

[2] Drew, R. I.., Proc. Phys. Soc. (London), B, 64, 1005 (1951).

[3] Brown, D., Proc. Phys. Soc. (London), B, 6\%, 232 (1954).

[4] Brown, D. S., J. Sci. Instr., 32, 137 (April 1955).

[5] Saunders, J. B., J. Res. NBS 65B (Mathematics and Mathematical Physics), No. 4 (Oct-Dec 1961).

\section{Errata-Reference [5]}

Change the term $\sum_{\sigma=0}^{\nu-1} \sigma$ in each equation, (8) and (11), to read $\sum_{\sigma=1}^{\nu} \sigma$. Change the third member of eq (12) to read $\delta_{1}+3 \delta_{2}+6 \delta_{3}+10 \delta_{4}+15 \delta_{5}+21 \delta_{6}+28 \delta_{7}=0$. Change the second member of eq (16) to read $14 \delta_{0}+11 \delta_{1}+9 \delta_{2}+8 \delta_{3}=0$.

[6] Candler, C., Modern Interferometers, p. 487, Hilger \& Watts Ltd. (1951).

[7] Jenkins, F. A., and White, H. E. Fundamentals of Optics, p. 241, McGraw-Hill Book Co., Inc. (1957).

[8] Saunders, J. B., Construction of a Koesters double-image prism, J. Res. NBS 58, 21 (1957) RP2729.

[9] Milne-Thompson, L. M., The Calculus of Finite Differences, p. 2, Macmillan and Co., Ltd., St. Martin's Street, London (1951). 